\title{
TOEPLITZ OPERATORS IN TQFT VIA SKEIN THEORY
}

\author{
JULIEN MARCHÉ AND THIERRY PAUL
}

\begin{abstract}
Topological quantum field theory associates to a punctured surface $\Sigma$, a level $r$ and colors $c$ in $\{1, \ldots, r-1\}$ at the marked points a finite dimensional Hermitian space $V_{r}(\Sigma, c)$. Curves $\gamma$ on $\Sigma$ act as Hermitian operator $T_{r}^{\gamma}$ on these spaces. In the case of the punctured torus and the 4-times punctured sphere, we prove that the matrix elements of $T_{r}^{\gamma}$ have an asymptotic expansion in powers of $\frac{1}{r}$ and we identify the two first terms using trace functions on representation spaces of the surface in $\mathrm{SU}_{2}$. We conjecture a formula for the general case. Then we show that the curve operators are Toeplitz operators on the sphere in the sense that $T_{r}^{\gamma}=\Pi_{r} f_{r}^{\gamma} \Pi_{r}$ where $\Pi_{r}$ is the Toeplitz projector and $f_{r}^{\gamma}$ is an explicit function on the sphere which is smooth away from the poles. Using this formula, we show that under some assumptions on the colors associated to the marked points, the sequence $T_{r}^{\gamma}$ is a Toeplitz operator in the usual sense with principal symbol equal to the trace function and with subleading term explicitly computed. We use this result and semiclassical analysis in order to compute the asymptotics of matrix elements of the representation of the mapping class group of $\Sigma$ on $V_{r}(\Sigma, c)$. We recover in this way the result of Taylor and Woodward on the asymptotics of the quantum $6 \mathrm{j}$-symbols and treat the case of the punctured S-matrix.
\end{abstract}

\section{Contents}

1. Introduction and main results of the paper

2. Structure of curve operators in TQFT

3. Small genus cases

4. Curve operators as Toeplitz operators

5. Asymptotics of mapping group representations

Appendix A. Computations with fusion rules

\section{INTRODUCTION AND MAIN RESUlts OF THE PAPER}

Topological quantum field theory (TQFT) was introduced by E. Witten in 1989 as a physical model for the Jones polynomial of knots (see [W89]). Fix a compact group $G$, and a representation $V$ of $G$. He defined for any knot $K$ in a 3-manifold $M$ a partition function $Z_{r}(M, K)$ as a Feynman integral over all connections $A$ on some $G$-bundle over $M$ of the form

$$
Z_{r}(M, K)=\int \operatorname{Tr}_{V}\left(\operatorname{Hol}_{K} A\right) e^{i r \operatorname{CS}(A)} \mathrm{d} A .
$$

Received by the editors November 2, 2012 and, in revised form, September 17, 2013.

2010 Mathematics Subject Classification. Primary 47B35, 57M27.

The first author was supported by the Agence Nationale de la Recherche ANR-08-JCJC-011401 and the second is a member of the CNRS. 
In this formula, $\operatorname{Hol}_{K}(A)$ is the holonomy of the connection $A$ along $K$ and $\operatorname{CS}(A)$ is the Chern-Simons functional.

The fact that this invariant is indeed computable comes from cut-and-paste rules implied by formal properties of Feynman integration. It allowed E. Witten to recognize (an evaluation of) the Jones polynomial(s) of $K$ and also to predict the asymptotics of such invariants when $r$ goes to infinity. If the semi-classical parameter $r$ goes to infinity in the formula for $Z_{r}(M, K)$, the stationary phase principle implies that the integral should concentrate on critical points of the ChernSimons functional, that is, connections which are flat on $M \backslash K$. Moreover, if $M$ has boundary $\Sigma$, then $Z_{r}(M, K)$ should be interpreted as an element of the geometric quantization of the moduli space $\mathcal{M}(\Sigma, G)$ that is gauge equivalence classes of flat $G$-connections on $\Sigma$.

As far as we know, since then, there is no rigorous geometric construction of this TQFT. The states are non-abelian theta functions, but the association of a theta function to a manifold is not understood. On the other hand, N. Reshetikhin and V. Turaev developed in RT91 a rigorous combinatorial construction of the TQFT. We will use in this article the version of C. Blanchet, N. Habegger, G. Masbaum and P. Vogel ([BHMV] $)$ which works for $G=\mathrm{SU}_{2}$ and relies only on the combinatorics of the Kauffman bracket. The price to pay for these combinatorial constructions is that the geometry gets hidden, in particular, the natural expectations for the semi-classical limit $r \rightarrow \infty$ become very mysterious.

Here we study curve operators, that is, the natural action of curves on $\Sigma$ on the TQFT vector space associated to $\Sigma$, in the cases where $\Sigma$ is either a once punctured torus or a 4-times punctured sphere. In the case of the torus without punctures, the complete geometric picture has been understood by R. Gelca and A. Uribe GU03, G06.

Notice that in the present paper we use the combinatorial curve operators, as in [MN08, and not the geometric ones as in A06, A10, A11 which are by definition Toeplitz operators. In this sense, our settings differs from Andersen's. He uses complex structures on $\mathcal{M}(\Sigma, G)$ parametrized by the Teichmüller space of $\Sigma$ and defines curve operators by Toeplitz quantization of the trace functions, therefore not as they are defined in combinatorial TQFT. In the present paper we identify the matrix in an explicit basis of any curve operator defined in the framework of combinatorial TQFT with a Toeplitz operator on symplectic manifold with explicit complex structure, that we identify a posteriori with the moduli space $\mathcal{M}(\Sigma, G)$. We develop a method of computing the Toeplitz multiplier and show that it differs from the negative trace function by a term whose leading part is computed.

1.1. Main results. Let $\Sigma$ be either a once punctured torus or a 4-times punctured sphere. Then, under generic assumptions on the holonomy $t$ around the marked points, the moduli spaces $\mathcal{M}(\Sigma, t)$ (see Subsection 2.2) are symplectomorphic to the standard sphere $S^{2}=\mathbb{C} P^{1}$. Let $V_{r}(\Sigma, c)$ be the TQFT vector space associated to $\Sigma$ with level $r$ and colors $c$ at marked points (see Subsection 2.1). Any curve $\gamma$ on $\Sigma$ acts on $V_{r}(\Sigma, c)$ as a Hermitian operator $T_{r}^{\gamma}$. We will define in Subsection 4.2 .2 a sequence of colorings $c_{r}$ such that $\pi \frac{c_{r}}{r}$ converges to $t$ and the dimension of $V_{r}\left(\Sigma, c_{r}\right)$, denoted by $N$, grows linearly with $r$. One of the main goals of the paper is to realize any curve operator as a Toeplitz operator, once again in the case of the once punctured torus or the 4-times punctured sphere. 
Result 1 (see Theorem 4.12 in Section 4.2.2). Suppose that $\mathcal{M}(\Sigma, t)$ is smooth. Then there is a canonical diffeomorphism $\mathcal{M}(\Sigma, t) \simeq \mathbb{C} P^{1}$ such that for any curve $\gamma$ on $\Sigma$, the sequence of matrices $\left(T_{r}^{\gamma}\right)$ are Toeplitz operators with symbol $\sigma^{\gamma}=$ $\sigma_{0}^{\gamma}+\frac{1}{N} \sigma_{1}^{\gamma}+O\left(N^{-2}\right)$ with

$$
\begin{gathered}
\sigma_{0}^{\gamma}(\rho)=-\operatorname{Tr} \rho(\gamma), \\
\sigma_{1}^{\gamma}=\frac{1}{2} \Delta_{S} \sigma_{0}^{\gamma},
\end{gathered}
$$

where $\Delta_{S}$ is the Laplacian on the sphere which is equal to $\left(1+|z|^{2}\right)^{2} \partial_{z} \partial_{\bar{z}}$ in the canonical holomorphic coordinate $z$.

Let us remark that, though there is no Weyl quantization on the sphere, the condition (2) corresponds, on the flat case, to having no Weyl subsymbol.

In fact we prove a somehow more precise result which does not require any smoothness assumption. We prove the existence of an exact (non-semi-classical) symbol:

Result 2 (see Theorem 4.7 in Section 4.2). For a holonomy $t$ around the marked points satisfying some mild assumptions, any level $r \in \mathbb{N}$ big enough and any curve $\gamma \subset \Sigma$, there exist a function $f_{\gamma}^{r}$ on the sphere, smooth except possibly at the two poles, such that

$$
T_{r}^{\gamma}=\mathcal{T}_{r}^{f_{\gamma}^{r}}
$$

where $\mathcal{T}_{r}^{f}$ is the Toeplitz quantization, namely $\mathcal{T}_{r}^{f}=\Pi_{r} f \Pi_{r}$ and $\Pi_{r}$ is the Toeplitz projector.

Moreover

$$
f_{\gamma}^{r} \sim-\operatorname{Tr} \rho(\gamma)+\frac{1}{2 N} \Delta_{S} \sigma_{0}^{\gamma}+\sum_{k=2}^{\infty} N^{-k} \sigma_{k}^{\gamma},
$$

with $\sigma_{k}^{\gamma}$ smooth away from the poles and where $N=\operatorname{dim} V_{r}(\Sigma, c)$.

Let us remark that this result gives a Toeplitz framework for curve operators even in the singular case, namely the case where the trace function is not smooth. In this case, standard asymptotic methods will fail to give a Toeplitz symbol by iterations.

The proof of this theorem goes through a closed formula relating the exact symbol of a Toeplitz operator to its matrix elements on the canonical basis. We first prove the following theorem:

Result 3 (see Theorem 2.5 in Section 2). For any curve $\gamma \subset \Sigma$ there exists a non-negative integer $k$ such that if we denote by $F_{n, m}^{\gamma}$ the matrix elements of $T_{r}^{\gamma}$ in the canonical basis of $V_{r}(\Sigma, c)$, then

$$
F_{n, m}=0 \quad \text { if }|n-m|>k .
$$

Let $\tau \in[0,1]$ and $\theta \in \mathbb{R} / 2 \pi \mathbb{Z}$ be cylindrical coordinates on $S^{2} \simeq \mathcal{M}(\Sigma, t)$.

Result 4 (see Theorem 2.5 in Section 2). There exist a sequence of $C^{\infty}$ functions $F(\tau, \theta, r)=\sum_{|\mu| \leq k} F_{\mu}(\tau, r) e^{i \mu \theta}$ on the sphere satisfying for $0<\tau<1$,

$$
F(\tau, \theta, r)=F(\tau, \theta, \infty)+\frac{1}{2 i r} \partial_{\tau} \partial_{\theta} F(\tau, \theta, \infty)+O\left(r^{-2}\right)
$$


with $F(\tau, \theta, \infty)=-\operatorname{Tr} \rho(\gamma)$ where $\rho$ is parametrized by $(\tau, \theta)$ and such that the matrix elements $F_{n, m}$ of $T_{r}^{\gamma}$ on the canonical basis are for $0<\frac{n}{r}<1$,

$$
F_{n, n+\mu}=F_{\mu}\left(\frac{n}{r}, r\right) .
$$

We also conjecture in Section 2 these two last results to be true in the higher genus case.

The curve operators $T_{r}^{\gamma}$ play a crucial role in understanding the asymptotic properties of TQFT. They were the key ingredient for proving the asymptotic faithfulness of the quantum representations; see A06, FWW, MN08. In the case of the torus, they are used extensively in CM11a, CM11b. Their spectral decomposition is directly linked to the basis coming from the combinatorial TQFT and their symbol and sub-symbol give the semi-classical properties at first order. As an application of Results 1 and 4, we can use standard techniques from semi-classical analysis to compute the asymptotics of the pairings between different basis element, recovering the quantum 6j-symbols computed in [TW05] (see Section 5.2.1) and as a new example, the S-matrix of the punctured torus which is detailed below. Let us remark that the condition (2), which determines the sub-principal contribution of the Toeplitz symbol out of the classical trace function (1) enters at leading orders in these asymptotics and is somehow crucial in order to get topological invariant terms.

Notice that the case of classical $6 \mathrm{j}$-symbols were treated by a similar method in [C10.

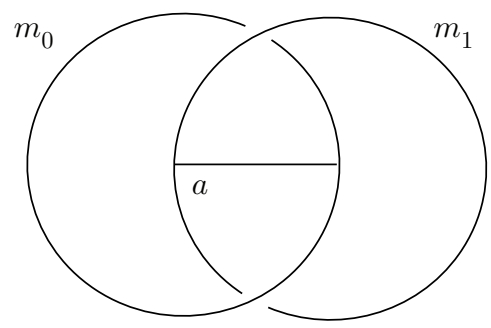

Figure 1. Punctured S-matrix

Let $\Gamma$ be the graph shown in Figure 1 colored by $c=\left(m_{0}, m_{1}, a\right)$ where $a$ and $D$ are odd integers satisfying $\frac{a}{2}<m_{i}<D-\frac{a}{2}$ for $i=0,1$. We denote by $\langle\Gamma, c\rangle_{r}$ the evaluation of the Kauffman bracket of the colored graph $(\Gamma, c)$ at $t=-e^{i \pi / 2 r}$; see Subsection 2.5. Then we have the following result (see Proposition [5.8):

Result 5. For any odd $\bar{r}$, setting $r=D \bar{r}$ one has

$$
\langle\Gamma, \bar{r} c\rangle_{r}=\frac{2 r}{\pi} N_{r}\left(G^{-1 / 4} \cos \left(\frac{r}{2 \pi} S+\frac{\pi}{4}\right)+O\left(r^{-1}\right)\right),
$$

where

$$
\begin{aligned}
& -N_{r}=\frac{\left(\left\langle m_{0}+\frac{a-1}{2}\right\rangle !\left\langle m_{0}-\frac{a+1}{2}\right\rangle !\left\langle m_{1}+\frac{a-1}{2}\right\rangle !\left\langle m_{1}-\frac{a+1}{2}\right\rangle !\right)^{1 / 2}\left\langle\frac{a-1}{2}\right\rangle !^{2}}{\langle a-1\rangle !\left\langle m_{0}-1\right\rangle !\left\langle m_{1}-1\right\rangle !}, \\
& \text { - }\langle n\rangle=\sin \left(\frac{\pi n}{r}\right) \text { and }\langle n\rangle !=\prod_{k=1}^{n}\langle k\rangle, \\
& \text { - } G=\cos \left(\frac{\alpha}{2}\right)^{2}-\cos \left(\tau_{0}\right)^{2}-\cos \left(\tau_{1}\right)^{2}-\cos \left(\tau_{0}\right)^{2} \cos \left(\tau_{1}\right)^{2}, \\
& \text { - } \alpha=\frac{\pi a}{D}, \tau_{0}=\frac{\pi m_{0}}{D}, \tau_{1}=\frac{\pi m_{1}}{D}, \\
& \text { - } S \text { is the area of the moduli space }\left\{(A, B) \in \mathrm{SU}_{2}, \operatorname{Tr}\left(A B A^{-1} B^{-1}\right)=2 \cos (\alpha),\right. \\
& \left.\quad \operatorname{Tr}(A) \leq 2 \cos \left(\tau_{0}\right), \operatorname{Tr}(B) \leq 2 \cos \left(\tau_{1}\right)\right\} / \sim .
\end{aligned}
$$


Finally let us mention that we proved in [MP11] some partial results concerning the case of a surface of genus 2. The moduli space $\mathcal{M}(\Sigma, G)$ is known to be isomorphic to $\mathbb{P}^{3}$ with a singular symplectic structure; see Hu98. Therefore its geometric quantization is not standardly well defined. Nevertheless, we prove that some curve operators are on the one side Toeplitz operators acting on the standard quantization of $\mathbb{P}^{3}$, and on the other side, their leading symbol is equal in a dense open set of $\mathcal{M}(\Sigma, G)$ to the corresponding trace functions.

Consider the three curves $\gamma, \delta, \eta$ of Figure 2.

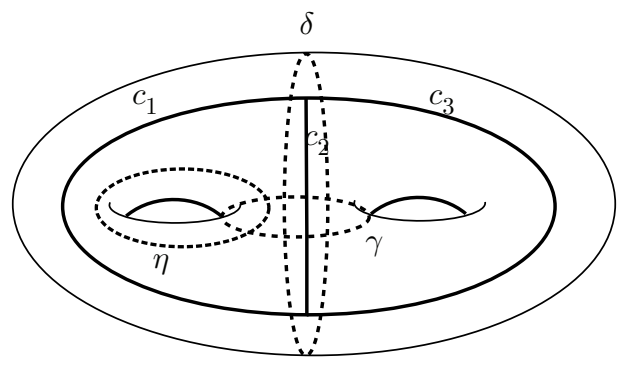

Figure 2. Curves on a genus 2 surface

Result 6 (see Theorem 6.3 in [MP11]). $T_{r}^{\gamma}, T_{r}^{\delta}, T_{r}^{\eta}$ are Toeplitz operators with exact symbols in the sense of Result $2, f_{\gamma}^{r}, f_{\delta}^{r}, f_{\eta}^{r}$ defined on $\mathbb{P}^{3}$, admitting a smooth asymptotic expansion in $\mathbb{P}^{3} /\left\{z_{0} z_{1} z_{2} z_{3}=0\right\}$. Moreover $\sigma_{0}^{\gamma}=-\operatorname{Tr} \rho(\gamma)$ and $\sigma_{0}^{\delta}=-\operatorname{Tr} \rho(\delta)$.

We believe that the methods developed in the present paper can be generalized to the higher genus cases, using the geometric quantization of toric manifolds and the Mellin transform strategy of Section 4 .

Conjecture 1. Result 2 is true in the general higher genus case by replacing the sphere by a toric manifold modeling the moduli space $\mathcal{M}(\Sigma, t)$, the two poles by the singularities of $\mathcal{M}(\Sigma, t)$ and the Laplacian on the sphere by the one of $\mathcal{M}(\Sigma, t)$.

1.2. Organization of the paper. Section 2 is devoted to the general structure of curve operators. The preliminary considerations on TQFT exposed in the beginning of the section lead to the definition of trigonometric operators that we conjecture to be the shape of general curve operators, together with the "sub-principal symbol" property (5). We prove that the conjecture is true in the case of the punctured torus and the 4 -times punctured sphere in Section 3 by first computing the matrix of three particular curve operators. By relying heavily on the properties of the Kauffman algebra, we then show that the latter computation is sufficient to prove the statement for any curve in these two punctured surfaces.

Section 4 is somehow the heart of the paper and contains the results on the Toeplitz structure of curve operators. After deriving an expression for the total symbol of a Toeplitz operator out of its matrix elements on a canonical basis, we show that the machinery applies to the case of any curve operator, even in the singular cases. We also compute the asymptotic regime and show that the total symbol is a classical one in the regular case.

Section 5 is devoted to the computation of pairing formulas, namely basis change matrices. We obtain a general asymptotic formula for the matrix elements of the 
quantum representations of the mapping class group of the two punctured surfaces. In particular we recover, by pure semi-classical methods, the asymptotic of $6 j$ symbols and derive the case of the punctured S-matrix.

\section{Structure of CURVE operators in TQFT}

2.1. Basics of TQFT. Let $\Sigma$ be a closed oriented surface with marked points $p_{1}, \ldots, p_{n}$. Fix an integer $r>0$ and let $\mathcal{C}_{r}=\{1, \ldots, r-1\}$ be the set of colors. Given a coloring $c=\left(c_{1}, \ldots, c_{n}\right) \in \mathcal{C}_{r}^{n}$ of the marked points, the construction of BHMV gives a finite dimensional Hermitian vector space $V_{r}(\Sigma, c)$. In their notation, $p=2 r, A=-\exp (i \pi / 2 r)$ (notice that we shifted by 1 the colors). One can construct a basis of this space by considering a banded graph $\Gamma$ that is a finite graph with $n$ univalent vertices labeled by $p_{1}, \ldots, p_{n}$ and trivalent vertices with local orientations around vertices. This datum allows us to construct a surface $S$ which retracts on $\Gamma$ and such that the univalent vertices belong to the boundary of $S$.

Let $H$ be the 3 -manifold $S \times[0,1]$. A presentation of $\Sigma$ is a homeomorphism $h: \partial H \rightarrow \Sigma$ which respects orientation and such that for all $i, h\left(p_{i} \times\{1 / 2\}\right)=p_{i}$; see Figure 3

Let $E$ be the set of edges of $\Gamma$. We call admissible coloring of $\Gamma$ a map $\check{c}: E \rightarrow \mathcal{C}_{r}$ such that the following conditions hold:

- for each edge $e$ connected to a univalent vertex $p_{i}$ one has $\check{c}_{e}=c_{i}$,

- for any triple of edges $e, f, g$ adjacent to the same vertex one has

$$
\begin{gathered}
-\check{c}_{e}+\check{c}_{f}+\check{c}_{g} \text { is odd, } \\
-\quad \check{c}_{g}<\check{c}_{e}+\check{c}_{f}, \\
-\check{c}_{e}+\check{c}_{f}+\check{c}_{g}<2 r .
\end{gathered}
$$

The construction of [BHMV] provides for each admissible coloring $\check{c}$ a vector $\varphi_{\check{c}} \in$ $V_{r}(\Sigma, c)$ obtained by cabling the graph $\Gamma$ by a specific combination of multicurves. Moreover, the family $\left(\varphi_{\check{c}}\right)$ when $\check{c}$ runs over all admissible colorings is a Hermitian basis of $V_{r}(\Sigma, c)$. This construction will be sketched in Subsection 2.5.

2.2. Representation spaces in $\mathbf{S U}_{2}$. Fix as before a surface $\Sigma$ with marked points $p_{1}, \ldots, p_{n}$. Suppose that these points are colored by $\left(t_{1}, \ldots, t_{n}\right) \in(\mathbb{Q} \pi)^{n}$ and denote by $\gamma_{i}$ a curve going around $p_{i}$. We define the following moduli space:

$$
\mathcal{M}(\Sigma, t)=\left\{\rho: \pi_{1}\left(\Sigma \backslash\left\{p_{1}, \ldots, p_{n}\right\}\right) \rightarrow \mathrm{SU}_{2} \text { s.t. } \forall i, \operatorname{Tr} \rho\left(\gamma_{i}\right)=2 \cos \left(t_{i}\right)\right\} / \sim .
$$

One has $\rho \sim \rho^{\prime}$ if there is $g \in \mathrm{SU}_{2}$ such that $\rho^{\prime}=g \rho g^{-1}$.

This space is a compact symplectic variety. It is smooth for generic values of $t$.

Let $U$ be the set of all maps $\tau: E \rightarrow[0, \pi]$ such that for any edge $e$ adjacent to a marked point $p_{i}$ one has $\tau_{i}=t_{i}$ and for any triple of edges $e, f, g$ adjacent to the same vertex one has

$$
\begin{gathered}
-\tau_{g} \leq \tau_{e}+\tau_{f}, \\
-\tau_{e}+\tau_{f}+\tau_{g} \leq 2 \pi .
\end{gathered}
$$

Given a curve $\gamma$ in $\Sigma$, we define a function $h_{\gamma}: \mathcal{M}(\Sigma, t) \rightarrow[0, \pi]$ by the formula $h_{\gamma}(\rho)=\arccos \left(\frac{1}{2} \operatorname{Tr} \rho(\gamma)\right)$. Let $p: \mathcal{M}(\Sigma, t) \rightarrow U$ be the map defined by $p(\rho)_{e}=$ $h_{C_{e}}(\rho)$ where $C_{e}$ is the circle dual to $e$ in $\Sigma$. The map $p$ is a continuous surjective map which is a smooth Lagrangian fibration over the interior of $U$.

Moreover, there is a preferred section $s: U \rightarrow \mathcal{M}(\Sigma, t)$ defined in the following way: for any edge $e$ we define a circle $D_{e}$ distinct from $C_{e}$ : if the edge $e$ matches two distinct vertices in $\Gamma$, then $D_{e}$ goes along the edge $e$, cuts $C_{e}$ in two points and 

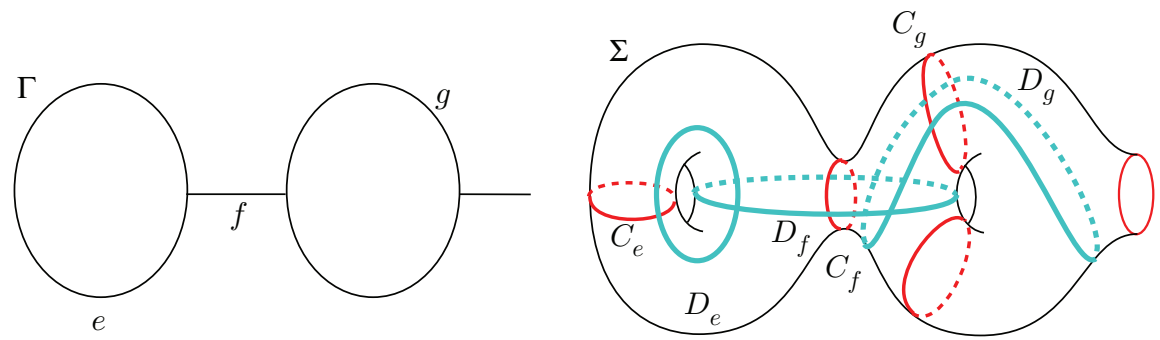

FiguRE 3. From a graph to a surface with a pants decomposition

no other circle $C_{f}$. If the edge $e$ joins a vertex to itself, then $D_{e}$ is the curve lying at the boundary of $S$ going along $e$. It cuts $C_{e}$ once and no other curve $C_{f}$. Some examples are shown in Figure 3 .

For $\theta_{e}$ in $S^{1}=\mathbb{R} / 2 \pi \mathbb{Z}$, we denote by $\theta_{e} . \rho$ the action on $\rho$ of the Hamiltonian flow of the map $h_{C_{e}}(\rho)=p(\rho)_{e}$.

Lemma 2.1. For any $\tau$ in the interior of $U$, there is a unique $\rho \in \mathcal{M}(\Sigma, t)$ which minimizes simultaneously the functions $h_{D_{e}}$ on the fiber of $p$.

Proof. By Goldman's formula, the Poisson bracket of two functions $h_{\delta}$ and $h_{\delta}$ vanishes if $\gamma$ and $\delta$ do not intersect (see [G86]). As $D_{e}$ intersects $C_{f}$ if and only if $e=f$, we see that all minimizations can be done independently on each edge. The proof follows by inspection of the two cases, that is, the 4-times-punctured sphere and the once-punctured torus which is done in Lemmas 3.2 and 3.5 .

2.3. The Kauffman algebra. For any oriented 3-manifold $M$, let $K(M, A)$ be the quotient of the free $\mathbb{C}\left[A^{ \pm 1}\right]$-module generated by isotopy classes of banded links in $M$ modulo the Kauffman relations shown in Figure 4.

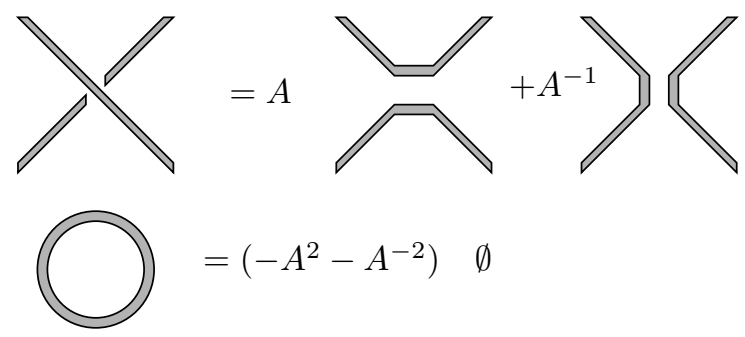

FiguRE 4. Kauffman relations

Write $K(\Sigma, A)=K\left(\left(\Sigma \backslash\left\{p_{1}, \ldots, p_{n}\right\}\right) \times[0,1], A\right)$. This module is an algebra where the product $\delta \cdot \gamma$ of two banded links is obtained by stretching $\delta$ and $\gamma$ so that they live respectively in $\Sigma \times[1 / 2,1]$ and $\Sigma \times[0,1 / 2]$ and then gluing the two intervals.

We call multicurve on $\Sigma$ any 1-submanifold avoiding the marked points such that no component bounds a disc avoiding the marked points. It is well known that $K(\Sigma, A)$ is a free $\mathbb{C}\left[A^{ \pm 1}\right]$-module generated by multicurves. Using this preferred basis, we identify $K(\Sigma, \zeta)=K(\Sigma, A) \otimes \mathbb{C}_{A=\zeta}$ with $K(\Sigma,-1)$ for any $\zeta \neq 0$ and we embed $K(\Sigma,-\exp (i \pi \hbar / 2))=K(\Sigma, A) \otimes \mathbb{C}[[h]]_{A=-\exp (i \pi \hbar / 2)}$ in $K(\Sigma,-1)[[\hbar]]$. 
We have the following theorem:

Theorem 2.2. The map $K(\Sigma,-1) \rightarrow C^{\infty}(\mathcal{M}(\Sigma, t))$ defined by $\gamma \mapsto f_{\gamma}$ where $f_{\gamma}(\rho)=-\operatorname{Tr} \rho(\gamma)$ is an injective morphism of algebras.

For any two multicurves $\gamma, \delta$ considered as elements of $K(\Sigma,-1)[[\hbar]]$, one has

$$
\gamma \cdot \delta=f_{\gamma} f_{\delta}+\frac{\hbar}{2 i}\left\{f_{\gamma}, f_{\delta}\right\}+o(\hbar) .
$$

For each $r>0$, the Kauffman algebra at $\zeta_{r}=-e^{i \pi / 2 r}$ acts on $V_{r}(\Sigma, c)$ where a curve $\gamma$ acts by a Hermitian operator $T_{r}^{\gamma}$ called a curve operator.

2.4. The curve operators in TQFT. In this section, we give some details on the skein construction of TQFT in order to explain the definition and computation of the curve operators.

Let $\Sigma$ be a closed surface and $H$ be a handlebody such that $\partial H=\Sigma$. Fix an integer $r$ and consider the Kauffman module $K\left(H, \zeta_{r}\right)$. For any embedding $j$ of $H$ in $S^{3}$, we define a subspace

$$
N_{r}^{j}=\left\{x \in K\left(H, \zeta_{r}\right), \forall y \in K\left(S^{3} \backslash j(H), \zeta_{r}\right), \quad\langle x, y\rangle=0 \in K\left(S^{3}, \zeta_{r}\right)\right\} .
$$

Suppose that a 3-manifold $M$ is the union of two 3-manifolds $M_{1} \cup M_{2}$. There is a natural pairing $K\left(M_{1}, A\right) \times K\left(M_{2}, A\right) \rightarrow K(M, A)$ defined barccosy sending two banded links $L_{1} \subset M_{1}, L_{2} \subset M_{2}$ to their union $L_{1} \amalg L_{2}$ viewed as a banded link in $M_{1} \cup M_{2}$. This is the meaning of the pairing in the formula above for $M_{1}=H, M_{2}=S^{3} \backslash j(H)$ and $M=S^{3}$.

The point is that $N_{r}^{j}$ is a subspace of finite codimension in $K\left(H, \zeta_{r}\right)$ which does not depend on $j$. We then set $V_{r}(\Sigma)=K\left(H, \zeta_{r}\right) / N_{r}^{j}$. Note that $V_{r}(\Sigma, c)$ is also known as the reduced Kauffman bracket skein module.

Given a embedding $j: H \rightarrow S^{3}$ it defines an embedding of $\Sigma=\partial H$. Thicken slightly its image in $S^{3}$ such that one has the decomposition

$$
H \cup \Sigma \times[0,1] \cup S^{3} \backslash j(H)=S^{3} .
$$

We deduce from it a natural map

$$
K\left(H, \zeta_{r}\right) \times K\left(\Sigma, \zeta_{r}\right) \times K\left(S^{3} \backslash j(H), \zeta_{r}\right) \rightarrow K\left(S^{3}, \zeta_{r}\right)=\mathbb{C}
$$

denoted by $(x, y, z) \mapsto\langle x|y| z\rangle$. If for any $z$ we have $\langle x|\emptyset| z\rangle=0$, then for any $y$ we also have $\langle x|y| z\rangle=0$ by "pushing $y$ to the right". It follows that "pushing $y$ to the left" defines an action of $K\left(\Sigma, \zeta_{r}\right)$ on $V_{r}(\Sigma)$.

2.5. Jones-Wenzl idempotents, colorings and marked points. In order to deal with the case when $\Gamma$ has boundary points and to construct the TQFT basis mentioned in Subsection 2.1, we need to introduce the Temperley-Lieb algebras $\mathcal{T}_{l}$ and the Jones-Wenzl idempotents $f_{l} \in \mathcal{T}_{l}$. Let $P_{l} \subset(0,1)$ be a finite set with $l$ elements and $\mathcal{T}_{l}(A)$ be the $\mathbb{C}\left[A^{ \pm 1}\right]$-module generated by banded tangles $L \subset[0,1]^{3}$ such that $\partial L=P_{l} \times\{1 / 2\} \times\{ \pm 1\}$ modulo Kauffman relations. The product $L_{1} \cdot L_{2}$ of two tangles is given by stacking $L_{1}$ above $L_{2}$.

One can define recursively the Jones-Wenzl idempotents by $f_{0}=0, f_{1}=1$ and the relations of Figure 5 where we set $[l]=\frac{A^{2 l}-A^{-2 l}}{A^{2}-A^{-2}}$. If $A=\zeta_{r}$, the idempotents $f_{l}$ are well defined in $\mathcal{T}_{r}$ provided that we have $l<r$.

Suppose now that $\Sigma$ is a surface with punctures $p_{1}, \ldots, p_{n}$. Fix a level $r$ and choose colors $c=\left(c_{1}, \ldots, c_{n}\right) \in \mathcal{C}_{r}^{n}$. We define $V_{r}(\Sigma, c)$ in the following way. Consider for all $i \in\{1, \ldots, n\}$ a subset $P_{i} \in \Sigma$ of cardinality $c_{i}-1$ and lying in 

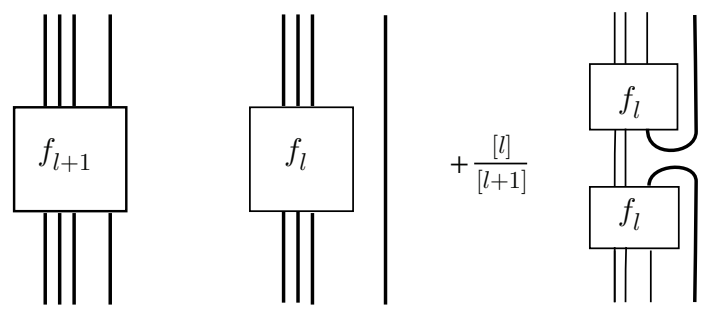

FiguRE 5. Recurrence relation for the Jones-Wenzl idempotents

a small neighborhood of $p_{i}$. We define the relative skein module $K(H, c, A)$ as the $\mathbb{C}\left[A^{ \pm 1}\right]$-module generated by banded tangles in $H$ whose intersection with $\Sigma$ is $\bigcup_{i} P_{i}$ modulo the Kauffman relations. Then for any embedding $j: H \rightarrow S^{3}$ we set

$$
N_{r}^{j}=\left\{x \in K\left(H, c, \zeta_{r}\right), \quad \forall z \in K\left(S^{3} \backslash j(H), c, \zeta_{r}\right) \quad\left\langle x\left|\bigotimes_{i=1}^{r-1} f_{c_{i}-1}\right| z\right\rangle=0\right\} .
$$

We set as before $V_{r}(\Sigma, c)=K\left(H, c, \zeta_{r}\right) / N_{r}^{j}$ and the curve operator $T_{r}^{\gamma}$ is defined in the same way provided that the curve $\gamma$ does not touch the punctures $p_{1}, \ldots, p_{n}$.

We are ready to define the basis of the latter space. Let $\Gamma$ be a trivalent graph encoding a pants decomposition of $\Sigma$ as in Subsection 2.1. Let $S$ be the surface containing $\Gamma$ and set $H=S \times[0,1]$. Given any admissible map $\check{c}: E \rightarrow \mathcal{C}_{r}$ we define $\psi_{\check{c}} \in K\left(H, c, \zeta_{r}\right)$ in the following way:

- Replace each edge $e$ of $\Gamma$ by $\check{c}_{e}-1$ parallel copies lying on $S$.

- Insert in the middle of each edge the idempotent $(-1)^{\check{c}_{e}-1} f_{\check{c}_{e}-1}$.

- In the neighborhood of each trivalent vertex, join the three bunches of lines in $S$ in the unique possible way avoiding crossings.

It happens that this family of vectors is an orthogonal basis of $V_{r}(\Sigma, c)$ for a natural Hermitian structure we do not define here. We refer to Theorem 4.11 in BHMV] for the proof and the following formula:

$$
\left\|\psi_{\breve{c}}\right\|^{2}=\left(\frac{2}{r}\right)^{\chi(\Gamma) / 2} \frac{\prod_{v}\left\langle c_{v}^{1}, c_{v}^{2}, c_{v}^{3}\right\rangle}{\prod_{e}\left\langle c_{e}\right\rangle}
$$

where for any trivalent vertex $v$ of $\Gamma$ denote by $c_{v}^{1}, c_{v}^{2}, c_{v}^{3}$ the colors of the edges incoming at $v$ and for any internal edge $e, c_{e}$ denotes the color of that edge. We also set $\langle n\rangle=\sin (\pi n / r),\langle n\rangle !=\prod_{k=1}^{n}\langle k\rangle$ and

$$
\langle a, b, c\rangle=\frac{\langle i+j+k+1\rangle !\langle i\rangle !\langle j\rangle !\langle k\rangle !}{\langle j+k\rangle !\langle i+k\rangle !\langle i+j\rangle !}
$$

for $i, j, k$ defined by $a=j+k+1, b=i+k+1$ and $c=i+j+1$. The vectors $\varphi_{\check{c}}$ used in this article are given by the formula $\varphi_{\check{c}}=\frac{\psi_{\check{c}}}{\left\|\psi_{\check{c}}\right\|}$.

2.6. Trigonometric operators. Let $t=\left(t_{1}, \ldots t_{n}\right)$ be a family in $([0, \pi] \cap \mathbb{Q} \pi)^{n}$. Let $D$ be the common denominator of the rational numbers $t_{j} / \pi$. We will denote by $c_{r}$ the sequence of colorings $c_{r}=\left(r \frac{t_{j}}{\pi}\right) \in \mathcal{C}_{r}^{n}$ and suppose implicitly that $r$ is a multiple of $D$ so that these colorings take integral values.

Definition 2.3. A family of operators $T_{r} \in \operatorname{End}\left(V_{r}\left(\Sigma, c_{r}\right)\right)$ is called trigonometric if there is an open subset $V \subset U \times[0,1]$ containing $\operatorname{Int}(U) \times\{0\}$, a finite family 
of smooth functions $F_{k}: V \rightarrow \mathbb{R}$ indexed by maps $k: E \rightarrow \mathbb{Z}$ such that for all admissible colorings $\check{c}$, one has $T_{r} \varphi_{\check{c}}=\sum_{k} F_{k}\left(\frac{\pi \check{c}}{r}, \frac{\pi}{r}\right) \varphi_{\check{c}+k}$.

Any multicurve $\gamma$ gives rise in TQFT to a family of operators $T_{r}^{\gamma} \in \operatorname{End}\left(V_{r}\left(\Sigma, c_{r}\right)\right)$ : these operators are called curve operators. We make the following conjecture:

Conjecture 2. For any multicurve $\gamma$, the curve operator $T_{r}^{\gamma}$ is trigonometric. The coefficients $F_{k}$ are recursively computable and verify the following properties:

(1) $F_{k}$ vanishes if for some edge $e$ the geometric intersection of $\gamma$ with $C_{e}$ is lower than $k_{e}$.

(2) The map $F_{k}(\cdot, 0): U \rightarrow \mathbb{R}$ is the $k$-th Fourier coefficient of the function $f_{\gamma}$ with respect to the action of $\left(S^{1}\right)^{E}$ described in G86, CM09.

Let $\sigma^{\gamma}=\sum_{k} F_{k}(\tau, \hbar) e^{i k \theta}$ where $\tau$ is an element of the interior of $U, \hbar$ is a real parameter, $\theta \in(\mathbb{R} / 2 \pi \mathbb{Z})^{E}, k \in \mathbb{Z}^{E}$ and $k \theta=\sum_{e} k_{e} \theta_{e}$.

Definition 2.4. $\sigma^{\gamma}$ is called the $\psi$-symbol of $T_{r}^{\gamma}$.

We remark that the data $(\tau, \theta)$ are action-angle coordinates on the open subset of $\mathcal{M}(\Sigma, t)$ defined as $p^{-1}(\operatorname{Int}(U))$. Hence, $\psi$-symbol $\sigma^{\gamma}$ may be interpreted as a deformation of the trace function $f_{\gamma}$. The terminology $\psi$-symbol stands for pseudodifferential operator.

The following conjecture gives the first order of the deformation:

Conjecture 3. For any multicurve $\gamma$, the $\psi$-symbol $\sigma^{\gamma}$ has the following asymptotic development:

$$
\sigma^{\gamma}=f_{\gamma}+\left(\frac{1}{2 i} \sum_{e} \frac{\partial^{2} f_{\gamma}}{\partial \theta_{e} \partial \tau_{e}}\right) \hbar+o(\hbar) .
$$

The statements of Conjecture 2 easily transfer to statements on the $\psi$-symbol $\sigma^{\gamma}$, hence the proof should consist of computing this symbol as precisely as possible. This conjecture is by no means inaccessible: we prove it for the case of the oncepunctured torus and the 4th-punctured sphere by analyzing three particular cases and using the structure of the Kauffman algebra on both surfaces (linked to $\operatorname{SL}(2, \mathbb{Z})$ and the Farey triangulation). We believe that a general proof will use a detailed study of the fractional Dehn twists on multicurves.

Theorem 2.5. Conjectures 2 and 3 hold if $\Sigma$ is a punctured torus or a 4-times punctured sphere.

\section{Small Genus CASES}

The proof of Theorem 2.5 will proceed from an explicit computation in two particular cases and various compatibilities with the Kauffman algebra. The easiest case is the punctured torus.

3.1. The once-punctured torus. Take $\Sigma$ a punctured torus, and $\Gamma$ the graph with one trivalent vertex and one univalent vertex as shown in Figure 6. We call $a \in \mathcal{C}_{r}$ the (odd) color of the marked point and $n$ the color of the loop $e$. Let $\gamma$ be the circle around the loop $\left(\gamma=C_{e}\right.$ in the notation of the preceding section) and $\delta$ the circle parallel to the loop $\left(\delta=D_{e}\right)$. 


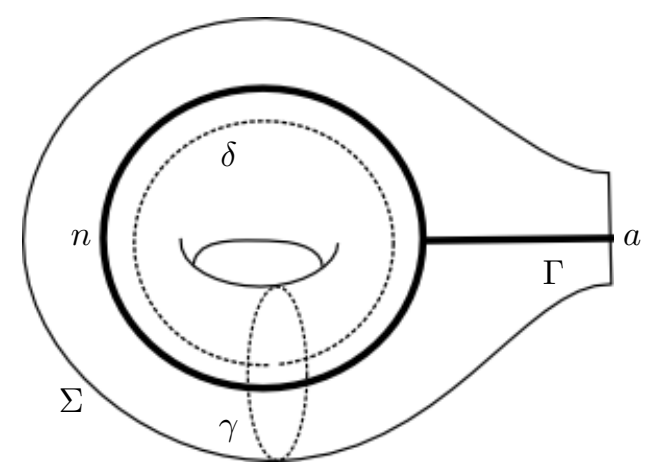

FiguRE 6. Basis for the punctured torus

Proposition 3.1. For $n$ satisfying $a / 2<n<r-a / 2$ one has:

$$
\begin{aligned}
T_{r}^{\gamma} \varphi_{n} & =-2 \cos \left(\frac{\pi n}{r}\right) \varphi_{n} \\
T_{r}^{\delta} \varphi_{n} & =u_{n+1} \varphi_{n+1}+u_{n} \varphi_{n-1} \text { where } \\
u_{n} & =-\left(\frac{\left\langle n+\frac{a-1}{2}\right\rangle\left\langle n-\frac{a+1}{2}\right\rangle}{\langle n\rangle\langle n-1\rangle}\right)^{1 / 2} .
\end{aligned}
$$

Proof. These formulas come from standard computations using fusion rules: the preceding section and Appendix $\mathrm{A}$ contain all the information needed to do the computation.

Let $\tau=\frac{\pi n}{r}, \alpha=\frac{\pi a}{r}$ and $\hbar=\frac{\pi}{r}$. Then, we compute directly from the above formula the $\psi$-symbols of $\gamma$ and $\delta$, namely $\sigma^{\gamma}=-2 \cos (\tau)$ and

$$
\begin{aligned}
\sigma^{\delta}= & -\left(\frac{\sin (\tau+\alpha / 2+\hbar / 2) \sin (\tau-\alpha / 2+\hbar / 2)}{\sin (\tau) \sin (\tau+\hbar)}\right)^{1 / 2} e^{i \theta} \\
& -\left(\frac{\sin (\tau+\alpha / 2-\hbar / 2) \sin (\tau-\alpha / 2-\hbar / 2)}{\sin (\tau) \sin (\tau-\hbar)}\right)^{1 / 2} e^{-i \theta} .
\end{aligned}
$$

The first point of Conjecture 2 is obviously satisfied as $\gamma$ and $\delta$ intersect $\gamma$ respectively 0 and 1 times. The second point is a consequence of the following lemma:

Lemma 3.2. Let $\Sigma$ be a punctured torus, $\alpha \in[0, \pi], \gamma$ and $\delta$ two curves on $\Sigma$ intersecting once. Let $(\tau, \theta)$ be the action-angle coordinates given in Lemma 2.1 and such that $\tau=h_{\gamma}$. Then, $f_{\delta}=-2 \frac{\sqrt{\sin (\tau+\alpha / 2) \sin (\tau-\alpha / 2)}}{\sin (\tau)} \cos (\theta)$.

Proof. Choose a base-point $x$ on the boundary of $\Sigma$ and represent $\gamma$ and $\delta$ as elements of $\pi_{1}(\Sigma, x)$. For any $\rho: \pi_{1}(\Sigma, x) \rightarrow \mathrm{SU}_{2}$, set $A=\rho(\gamma)$ and $B=\rho(\delta)$. The condition for $\rho$ to be in $\mathcal{M}(\Sigma, \alpha)$ is $\operatorname{Tr}\left(A B A^{-1} B^{-1}\right)=2 \cos (\alpha)$. For any $\theta \in \mathbb{R} / 2 \pi \mathbb{Z}$, set $U_{\theta}=\left(\begin{array}{cc}e^{i \theta} & 0 \\ 0 & e^{-i \theta}\end{array}\right)$. As $\operatorname{Tr}(A)=2 \cos (\tau)$, one can suppose up to conjugation that we have $A=U_{\tau}$. The Hamiltonian flow of $h_{\gamma}$ acts on $(A, B)$ 


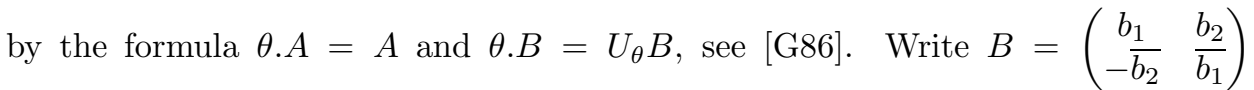
such that $\left|b_{1}\right|^{2}+\left|b_{2}\right|^{2}=1$. The formula $\operatorname{Tr}\left(A B A^{-1} B^{-1}\right)=2 \cos (\alpha)$ implies that $\left|b_{1}\right|^{2}=\frac{\cos (\alpha)-\cos (2 \tau)}{1-\cos (2 \tau)}=\frac{\sin (\tau+\alpha / 2) \sin (\tau-\alpha / 2)}{\sin (\tau)^{2}}$.

We also compute $\operatorname{Tr}(\theta . B)=2 \operatorname{Re}\left(b_{1} e^{i \theta}\right)=2 \frac{\sqrt{\sin (\tau+\alpha / 2) \sin (\tau-\alpha / 2)}}{\sin (\tau)} \cos (\theta+\psi)$ where $\psi=\arg \left(b_{1}\right)$. Recall that in Lemma 2.1 the origin for the angle $\theta$ is such that $\operatorname{Tr}(\theta . B)$ is maximal. Hence, one can suppose that $\psi=0$ which proves the lemma.

The geometric picture can be nicely visualized in the coordinates $x=\operatorname{Tr}(A), y=$ $\operatorname{Tr}(B), z=\operatorname{Tr}(A B)$. The moduli space $\mathcal{M}(\Sigma, \alpha)$ is isomorphic to the cubic $\{(x, y, z)$ $\left.\in[-2,2]^{3}, x^{2}+y^{2}+z^{2}-x y z-2=2 \cos (\alpha)\right\}$. The level sets of $x$ are ellipses on which the Hamiltonian flow $\theta$ acts by rotation. The level set $\theta=0$ is given by the maximum of $y$ on the level sets of $x$. It is then defined as the intersection of the cubic with the half plane $\{z=0, y \geq 0\}$.

Let $T w^{\gamma}$ be the Dehn twist along $\gamma$ and denote by $\xi$ the curve $T w^{\gamma}(\delta)$.

Lemma 3.3. For $\eta \in\{\gamma, \delta, \xi\}$, the following equation holds:

$$
\sigma^{\eta}=f_{\eta}+\left(\frac{1}{2 i} \frac{\partial^{2} f_{\eta}}{\partial \theta \partial \tau}\right) \hbar+o(\hbar)
$$

Proof. This is trivial for $\eta=\gamma$ as one has $\sigma^{\gamma}=f_{\gamma}$ and the first order term vanishes. For any $\eta$, set $\sigma^{\eta}=\sum_{k} F_{k}^{\eta}(\tau, \hbar) e^{i k \theta}$. The lemma is an immediate consequence of the following identity:

$$
\partial_{\hbar} F_{k}^{\eta}=\frac{k}{2} \partial_{\tau} F_{k}^{\eta}
$$

We check this identity by interpreting it as $\partial_{\hbar} F_{k}^{\eta}\left(\tau-\frac{k \hbar}{2}, \hbar\right)$. We compute $F_{1}^{\delta}(\tau-\hbar / 2, \hbar)=-\left(\frac{\sin (\tau+\alpha / 2) \sin (\tau-\alpha / 2)}{\sin (\tau-\hbar / 2) \sin (\tau+\hbar / 2)}\right)^{1 / 2}=F_{1}(\tau, 0)+o(\hbar)$. The same argument works for $F_{-1}^{\delta}$.

Denote by $T w_{r}^{\gamma}$ the action of $T w^{\gamma}$ on $V_{r}(\Sigma, a)$. Following [BHMV p. 913], the twist acts on $\varphi_{n}$ by the formula $T w_{r}^{\gamma} \varphi_{n}=e^{\frac{i \pi}{2 r}\left(n^{2}-1\right)} \varphi_{n}$. Hence, from the identity $T_{r}^{\xi}=T w_{r}^{\gamma} T_{r}^{\delta}\left(T w_{r}^{\gamma}\right)^{-1}$, we obtain, for $n_{n}, u_{n+1}$ as in Proposition 3.1. $T_{r}^{\xi} \varphi_{n}=$ $u_{n+1} e^{\frac{i \pi}{2 r}(2 n+1)} \varphi_{n+1}+u_{n} e^{\frac{i \pi}{2 r}(-2 n+1)} \varphi_{n-1}$ and

$$
\sigma^{\xi}=e^{i \hbar / 2} \sigma^{\delta}(\tau, \theta+\tau)
$$

When computing $\partial_{\hbar} \sigma^{\xi}$ and $\frac{1}{2 i} \partial_{\tau \theta} f_{\xi}$, we obtain the same result as before changing $\theta$ into $\theta+\tau$ plus a term $\frac{i \pi}{2}$. This extra term cancels with the term produced in the derivation, proving the formula for $\xi$.

3.2. The 4th-punctured sphere. Consider a 4-times punctured sphere $\Sigma$, and let $\Gamma$ be the graph of Figure 7 with two univalent vertices and four univalent vertices. We call $a, b, c, d$ the colors of the marked points and $n$ the color of the internal edge $e$. Let $\zeta=C_{e}$ and $\eta=D_{e}$.

Proposition 3.4. For $n$ satisfying

$$
\max (|a-d|,|b-c|)<n<\min (a+d, 2 r-a-d, b+c, 2 r-b-c)
$$




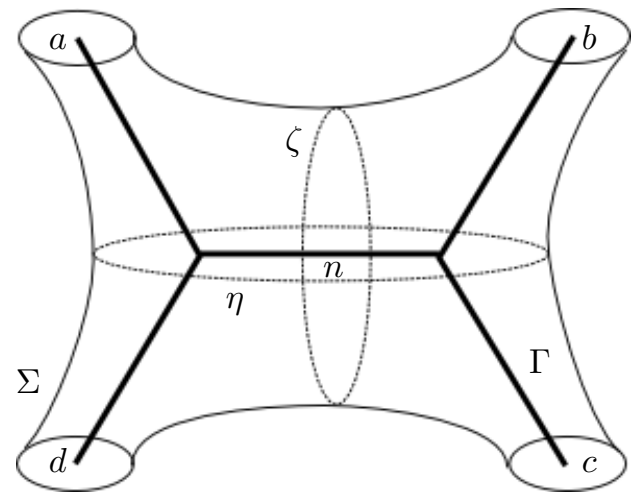

FiguRE 7. Basis for the punctured sphere

and $n+1=a+d=b+c \bmod 2$, one has:

$$
\begin{aligned}
T_{r}^{\zeta} \varphi_{n}= & -2 \cos \left(\frac{\pi n}{r}\right) \varphi_{n}, \\
T_{r}^{\eta} \varphi_{n}= & w_{n-2} \varphi_{n-2}+v_{n} \varphi_{n}+w_{n} \varphi_{n+2} \text { where } \\
w_{n}= & -4\left(\frac{\left\langle\frac{a+d-n-1}{2}\right\rangle\left\langle\frac{a-d+n+1}{2}\right\rangle\left\langle\frac{-a+d+n-1}{2}\right\rangle\left\langle\frac{a+d+n+1}{2}\right\rangle}{\langle n\rangle\langle n+1\rangle}\right. \\
& \left.\cdot \frac{\left\langle\frac{b+c-n-1}{2}\right\rangle\left\langle\frac{b-c+n+1}{2}\right\rangle\left\langle\frac{-b+c+n+1}{2}\right\rangle\left\langle\frac{b+c+n+1}{2}\right\rangle}{\langle n+1\rangle\langle n+2\rangle}\right)^{1 / 2}, \\
v_{n}= & -2 \cos \left(\frac{\pi(c+d-1)}{r}\right) \\
& -4 \frac{\left\langle\frac{a+d-n-1}{2}\right\rangle\left\langle\frac{a-d+n+1}{2}\right\rangle\left\langle\frac{b+c-n-1}{2}\right\rangle\left\langle\frac{b-c+n+1}{2}\right\rangle}{\langle n\rangle\langle n+1\rangle} \\
& -4 \frac{\left\langle\frac{a+d+n-1}{2}\right\rangle\left\langle\frac{-a+d+n-1}{2}\right\rangle\left\langle\frac{b+c+n-1}{2}\right\rangle\left\langle\frac{-b+c+n-1}{2}\right\rangle}{\langle n-1\rangle\langle n\rangle} .
\end{aligned}
$$

Proof. Again, we omit the proof since it is a long and standard computation using fusion rules; see Appendix $\mathrm{A}$.

Let $\tau=\frac{\pi n}{r}, \hbar=\frac{\pi}{r}, \alpha=\frac{\pi a}{r}, \beta=\frac{\pi b}{r}, \gamma=\frac{\pi c}{r}, \delta=\frac{\pi d}{r}$. Then, the $\psi$-symbols of $T^{\zeta}$ and $T^{\eta}$ are respectively $\sigma^{\gamma}=-2 \cos (\tau)$ and

$$
\begin{aligned}
\sigma^{\eta} & =-I(\tau, \hbar)-J(\tau, \hbar) e^{2 i \theta}-J(\tau-2 \hbar, \hbar) e^{-2 i \theta} \text { where } \\
I(\tau, \hbar)= & 2 \cos (\gamma+\delta-\hbar) \\
& +4 \frac{\sin \left(\frac{\alpha+\delta-\tau-\hbar}{2}\right) \sin \left(\frac{\alpha-\delta+\tau+\hbar}{2}\right) \sin \left(\frac{\beta+\gamma-\tau-\hbar}{2}\right) \sin \left(\frac{\beta-\gamma+\tau+\hbar}{2}\right)}{\sin (\tau) \sin (\tau+\hbar)} \\
& +4 \frac{\sin \left(\frac{\alpha+\delta+\tau-\hbar}{2}\right) \sin \left(\frac{-\alpha+\delta+\tau-\hbar}{2}\right) \sin \left(\frac{\beta+\gamma+\tau-\hbar}{2}\right) \sin \left(\frac{-\beta+\gamma+\tau-\hbar}{2}\right)}{\sin (\tau) \sin (\tau-\hbar)}
\end{aligned}
$$


and

$$
\begin{aligned}
J(\tau, \hbar)= & 4\left(\frac{\sin \left(\frac{\alpha+\delta-\tau-\hbar}{2}\right) \sin \left(\frac{\alpha-\delta+\tau+\hbar}{2}\right) \sin \left(\frac{-\alpha+\delta+\tau+\hbar}{2}\right) \sin \left(\frac{\alpha+\delta+\tau+\hbar}{2}\right)}{\sin (\tau) \sin (\tau+\hbar)}\right. \\
& \left.\cdot \frac{\sin \left(\frac{\beta+\gamma-\tau-\hbar}{2}\right) \sin \left(\frac{\beta-\gamma+\tau+\hbar}{2}\right) \sin \left(\frac{-\beta+\gamma+\tau+\hbar}{2}\right) \sin \left(\frac{\beta+\gamma+\tau+\hbar}{2}\right)}{\sin (\tau+\hbar) \sin (\tau+2 \hbar)}\right)^{1 / 2} .
\end{aligned}
$$

As for the case of the punctured torus, the first point of Conjecture 2 is trivially satisfied. We interpret the formulas where $\hbar=0$ in the following lemma.

Lemma 3.5. Fix $\alpha, \beta, \gamma, \delta \in[0, \pi]$ and let $\mathcal{M}=\left\{(A, B, C, D) \in \mathrm{SU}_{2}\right.$, s.t. $A B C D=1$, $\operatorname{Tr}(A)=2 \cos (\alpha), \operatorname{Tr}(B)=2 \cos (\beta), \operatorname{Tr}(C)=2 \cos (\gamma), \operatorname{Tr}(D)=2 \cos (\delta)\} / \sim$. Let $(\tau, \theta)$ be the action-angle coordinates on $\mathcal{M}$ given by Lemma 2.1 such that $\tau=h_{\zeta}$. Then, $f_{\eta}=\left.\sigma^{\eta}\right|_{\hbar=0}$.

This lemma uses the following easy sub-lemma which we do not prove.

Lemma 3.6. Let $A, B \in \mathrm{SU}_{2}$ be two matrices such that $A B=U_{\tau}$ for some $\tau \in$ $\mathbb{R} \backslash \pi \mathbb{Z}$. Then, the upper left entry of $A$ is $\frac{\cos (\alpha) \sin (\tau)+i(\cos (\beta)-\cos (\alpha) \cos (\tau))}{\sin (\tau)}$ where $\operatorname{Tr}(A)=2 \cos (\alpha)$ and $\operatorname{Tr}(B)=2 \cos (\beta)$.

Proof. One has $\operatorname{Tr}(A D)=2 \cos (\tau)$ and $\operatorname{Tr}(A B)=2 \cos \left(h_{\eta}\right)$. One can suppose up to conjugation that $D A=U_{\tau}$. Then, the Hamiltonian flow of $h_{\zeta}$ acts on the 4-tuple $(A, B, C, D)$ by $\theta \cdot(A, B, C, D)=\left(A, U_{\theta} B U_{-\theta}, U_{\theta} C U_{-\theta}, D\right)$. If one writes $A=\left(\begin{array}{cc}a_{1} & a_{2} \\ -\overline{a_{2}} & \overline{a_{1}}\end{array}\right)$ and $B=\left(\begin{array}{cc}b_{1} & b_{2} \\ -\overline{b_{2}} & \overline{b_{1}}\end{array}\right)$ such that $\left|a_{1}\right|^{2}+\left|a_{2}\right|^{2}=\left|b_{1}\right|^{2}+\left|b_{2}\right|^{2}=1$, we compute

$$
\operatorname{Tr}((\theta \cdot A)(\theta . B))=2 \operatorname{Re}\left(a_{1} b_{1}\right)-2 \operatorname{Re}\left(a_{2} \overline{b_{2}} e^{-2 i \theta}\right) .
$$

As $A^{-1} D^{-1}=U_{-\tau}$ and $B C=U_{-\tau}$, we find using the sub-lemma that $\overline{a_{1}}=$ $\frac{\cos (\alpha) \sin (\tau)+i(\cos (\alpha) \cos (\tau)-\cos (\delta))}{\sin (\tau)}$ and $b_{1}=\frac{\cos (\beta) \sin (\tau)+i(\cos (\beta) \cos (\tau)-\cos (\gamma))}{\sin (\tau)}$. We obtain by tedious trigonometric computations:

$$
\begin{aligned}
2 \operatorname{Re}\left(a_{1} b_{1}\right) & =\frac{2}{\sin (\tau)^{2}}(\cos (\alpha) \cos (\beta)+\cos (\gamma) \cos (\delta)-\cos (\tau)(\cos (\alpha) \cos (\gamma)+\cos (\beta) \cos (\gamma))) \\
& =I(\tau, 0) .
\end{aligned}
$$

We compute in the same way

$$
\begin{aligned}
\left|a_{2}\right|^{2} & =1-\left|a_{1}\right|^{2}=\frac{\sin (\tau)^{2}-\cos (\alpha)^{2}-\cos (\delta)^{2}+2 \cos (\alpha) \cos (\delta) \cos (\tau)}{\sin (\tau)^{2}} \\
& =\frac{-4 \sin \left(\frac{\alpha+\delta-\tau}{2}\right) \sin \left(\frac{\alpha-\delta+\tau}{2}\right) \sin \left(\frac{-\alpha+\delta+\tau}{2}\right) \sin \left(\frac{\alpha+\delta+\tau}{2}\right)}{\sin (\tau)^{2}} .
\end{aligned}
$$

Using this relation and the same one involving $\left|b_{2}\right|^{2}$, we find $\left|a_{2} b_{2}\right|=J(\tau, 0)$. Hence, we have $-f_{\eta}=I(\tau, 0)+2 J(\tau, 0) \cos (2 \theta+\psi)$. By the same argument as in Lemma 3.2. we know that $\theta$ is chosen such that $-f_{\eta}$ is maximal. Hence, one can suppose that $\psi=0$ which proves the lemma.

Again, there is a nice geometric picture of the preceding lemma. Consider the map $\mathcal{M} \rightarrow \mathrm{SU}_{2}^{4} / \mathrm{SU}_{2}$ which sends the 4-tuple $(A, B, C, D)$ to the 4-tuple $P_{1}=$ $1, P_{2}=A, P_{3}=A B, P_{4}=A B C$. Identifying $\mathrm{SU}_{2}$ with the sphere $S^{3}$, the distance between two elements $P$ and $Q$ is $\arccos \left(\frac{1}{2} \operatorname{Tr}\left(P Q^{-1}\right)\right)$. Hence, the distance $l_{i j}$ between $P_{i}$ and $P_{j}$ satisfies $l_{12}=\alpha, l_{23}=\beta, l_{34}=\gamma, l_{14}=\delta, l_{13}=\tau$. We may then interpret $\mathcal{M}$ as the moduli space of spherical quadrilaterals with fixed lengths. The 
coordinate $\tau$ is the length of the diagonal $P_{1} P_{3}$ whereas the angle $2 \theta$ is the dihedral angle at the edge $P_{1} P_{3}$. The coordinate $h_{\eta}$ is equal to the length $l_{24}$. For a fixed value of $\tau$, it is minimal for non-convex planar quadrilaterals. Hence, the level set $\theta=0$ consists of non-convex planar quadrilaterals whereas the level set $\theta=\pi / 2$ consists of convex planar quadrilaterals. Notice that in contrast with the case of the punctured torus, the angle coordinate is $\pi$-periodic: this is explained by the fact that the curve $\zeta$ is separating; see G86.

Let $\xi$ be the curve obtained by performing on $\eta$ a half Dehn twist along $\zeta$ as shown in Figure 8

Lemma 3.7. For $\lambda \in\{\zeta, \eta, \xi\}$, one has

$$
\sigma^{\lambda}=f_{\lambda}+\left(\frac{1}{2 i} \frac{\partial^{2} f_{\lambda}}{\partial \theta \partial \tau}\right) \hbar+o(\hbar)
$$

Proof. As for Lemma 3.3, the formula is trivial for the case $\lambda=\zeta$ and for the case $\lambda=\eta$, it will be a consequence of the equation

$$
F_{k}^{\eta}(\tau-k \hbar / 2, \hbar)=F_{k}^{\eta}(\tau, 0)+o(\hbar) .
$$

We check directly that one has $J(\tau-\hbar, \hbar)=o(\hbar)$ which implies that the above formula works for $k=2$ and $k=-2$. For $k=0$, one has to show $I(\tau, \hbar)=$ $I(\tau, 0)+o(\hbar)$. This was checked by a computation with the computer sofware Sage. The computation for $\xi$ is done in the same spirit as in Lemma 3.3 whereas instead of using the action of the twist, we use the half-twist coefficient

$$
H(c ; a, b)=(-1)^{\epsilon} \exp \left(\frac{i \pi}{4 r}\left(c^{2}-a^{2}-b^{2}+1\right)\right)
$$

where $\epsilon=\left(-a^{2}-b^{2}-c^{2}+2 a b+2 b c+2 a c-2 a-2 b-2 c+3\right) / 4$ (see [MV94]).
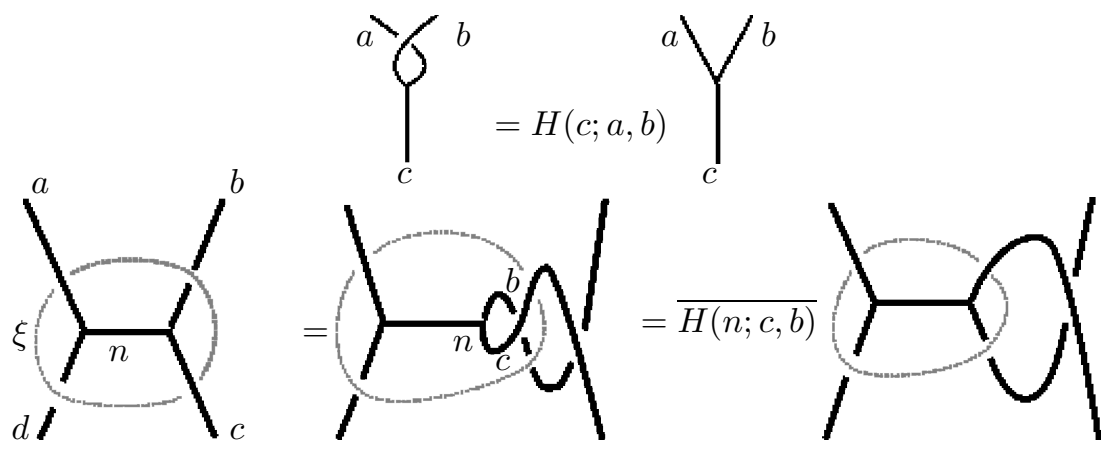

FiguRE 8. Half-twist acting on curves

In Figure 8, we observe that we can compute the operator $T_{r}^{\xi}$ knowing $T_{r}^{\eta}$ and formulas for the half-twist. We find precisely

$$
\begin{aligned}
T_{r}^{\xi} \varphi_{n} & =\frac{H(n-2 ; b, c)}{H(n ; c, b)} w_{n-2} \varphi_{n-2}+\frac{H(n ; b, c)}{H(n ; c, b)} v_{n} \varphi_{n}+\frac{H(n+2 ; b, c)}{H(n ; c, b)} w_{n} \varphi_{n+2} \\
& =-e^{i \pi(n-1) / r} w_{n-2} \varphi_{n-2}+v_{n} \varphi_{n}-e^{-i \pi(n+1) / r} w_{n} \varphi_{n+2} .
\end{aligned}
$$

Here, we used the formula for $w_{n}, v_{n}$ associated to the operator $T_{r}^{\eta}$ given in Proposition 3.4 where we implicitly inverted $b$ and $c$. From the last equation, we compute 
the symbol $\sigma^{\xi}=-e^{i(\tau-\hbar)} F_{-2}^{\eta} e^{-2 i \theta}+F_{0}^{\eta}-e^{-i(\tau+\hbar)} F_{2}^{\eta}$. The equation of the lemma is satisfied by a direct computation.

3.3. The general case. Consider a surface $\Sigma$ with marked points $p_{1}, \ldots, p_{n}$ colored by rational multiples of $\pi$ denoted by $t_{1}, \ldots, t_{n}$. Let $\Gamma$ be a graph associated to a pants decomposition of $\Sigma$.

Definition 3.8. We say that a multicurve $\gamma$ satisfies the property $(*)$ if

(1) $T_{r}^{\gamma}$ is a trigonometric operator (denote by $\sigma^{\gamma}$ its $\psi$-symbol).

(2) $\sigma^{\gamma}=f_{\gamma}+\left(\frac{1}{2 i} \sum_{e} \frac{\partial^{2} f_{\gamma}}{\partial \theta_{e} \partial \tau_{e}}\right) \hbar+o(\hbar)$.

We extend this definition to $K(\Sigma,-1)[[\hbar]]$ by $\mathbb{C}[[\hbar]]$-linearity.

Proposition 3.9. Let $\gamma$ and $\delta$ be two multicurves satisfying $(*)$; then their product in $K\left(\Sigma,-e^{i \pi \hbar / 2}\right)$ also satisfies $(*)$.

Proof. Let $\sigma^{\gamma}=\sum_{k} F_{k}(\tau, \hbar) e^{i k \theta}$ and $\sigma^{\delta}=\sum_{l} G_{l}(\tau, \hbar) e^{i l \theta}$ be the $\psi$-symbols of $T_{r}^{\gamma}$ and $T_{r}^{\delta}$. A direct computation shows that the product of the two operators is trigonometric with the following $\psi$-symbol:

$$
\sigma^{\gamma} \star \sigma^{\delta}=\sum_{n} e^{i n \theta} \sum_{k+l=n} F_{k}(\tau, \hbar) G_{l}(\tau+k \hbar, \hbar) .
$$

We compute the product $\gamma \cdot \delta$ in the Kauffman algebra by stacking the two multicurves and smoothing all crossings. As the map $\gamma \mapsto T_{r}^{\gamma}$ is a morphism from $K\left(\Sigma, \zeta_{r}\right)$ to $\operatorname{End}\left(V_{r}(\Sigma, r t / \pi)\right)$, we obtain the first point of the proposition.

Letting $\hbar$ go to 0 in the formula (9), we get the following expansion:

$$
\sigma^{\gamma} \star \sigma^{\delta}=\sigma^{\gamma} \sigma^{\delta}+\frac{\hbar}{i} \partial_{\theta} \sigma^{\gamma} \partial_{\tau} \sigma^{\delta}+o(\hbar) .
$$

On the other hand, as an element of $K(\Sigma,-1)[[\hbar]]$, we have $\gamma \cdot \delta=f_{\gamma} f_{\delta}+\frac{\hbar}{2 i}\left\{f_{\gamma}, f_{\delta}\right\}$; see [TU91]. Hence, we see that the $\psi$-symbols coincide at 0 -th order on $\hbar$. To equal the first order terms, one needs to show the following:

$$
f_{\gamma}\left(\frac{1}{2 i} \partial_{\theta} \partial_{\tau} f_{\delta}\right)+f_{\delta}\left(\frac{1}{2 i} \partial_{\theta} \partial_{\tau} f_{\gamma}\right)+\frac{1}{i} \partial_{\theta} f_{\gamma} \partial_{\tau} f_{\delta}=\frac{1}{2 i} \partial_{\theta} \partial \tau\left(f_{\gamma} f_{\delta}\right)+\frac{1}{2 i}\left\{f_{\gamma}, f_{\delta}\right\} .
$$

This formula holds thanks to the Leibniz formula and the fact that $(\theta, \tau)$ are action angle coordinates, meaning that $\left\{f_{\gamma}, f_{\delta}\right\}=\partial_{\theta} f_{\gamma} \partial_{\tau} f_{\delta}-\partial_{\tau} f_{\gamma} \partial_{\theta} f_{\delta}$.

We are now able to prove Theorem 2.5 by using the following results on the Kauffman algebra of the 1-torus and 4-sphere which are taken from [BP00].

Consider the Farey triangulation of the hyperbolic disc $\mathbb{H}$. We identify the boundary of $\mathbb{H}$ with $\mathbb{P}^{1}(\mathbb{R})$ and consider the set of rational points $\mathbb{P}^{1}(\mathbb{Q})$ in the boundary. A point $[a, b]$ corresponds to the simple closed curve in the punctured torus with slope $a / b$ (unique up to isotopy). The number of intersection points between two curves $\left[a_{1}, b_{1}\right]$ and $\left[a_{2}, b_{2}\right]$ is $\left|a_{1} b_{2}-a_{2} b_{1}\right|$. Join two points by a geodesic in $\mathbb{H}$ if and only if the corresponding curves intersect in one point. We get the Farey triangulation as shown in Figure 9

Consider two simple closed curves $\gamma_{1}, \gamma_{2}$ on the punctured torus $\Sigma$ which intersect once. Then stacking them in the Kauffman algebra $K(\Sigma, A)$ and smoothing the crossing we get $A \delta_{1}+A^{-1} \delta_{2}$ where $\delta_{1}$ and $\delta_{2}$ are the two curves obtained by 


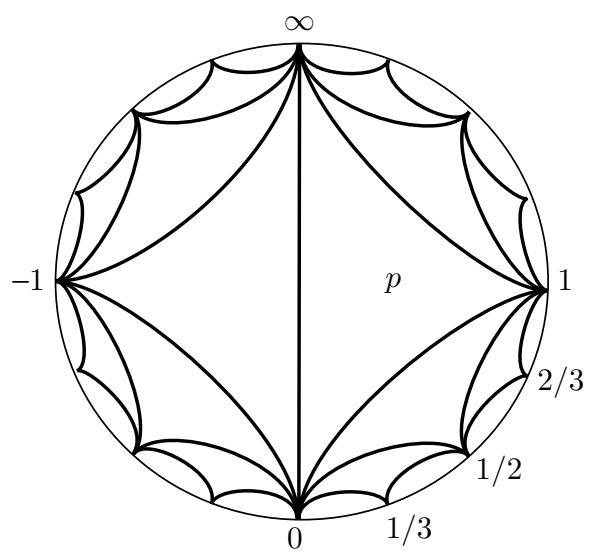

FiguRE 9. The Farey triangulation

smoothing $\gamma_{1} \cup \gamma_{2}$. On the Farey triangulation, we simply read that the product of two curves at the boundaries of an edge is a weighted sum of the two curves which are the vertices of the two triangles sharing the original edge. This immediately shows that the curves associated to the vertices of a fixed triangle generate the Kauffman algebra.

Consider the curves $\gamma, \delta$ and $\xi$ given in Subsection 3.1. Suppose that they correspond to $\infty, 0$ and 1 respectively in the Farey triangulation. For any curve $\eta$ such that $T_{r}^{\eta}$ is trigonometric, we define its degree as the highest $k \geq 0$ such that $F_{k}^{\eta}$ or $F_{-k}^{\eta}$ is non-zero: it is also equal to the number of non-vanishing diagonals of the matrix of $T_{r}^{\eta}$. Then we finally prove Theorem 2.5] in the case of the punctured torus.

Theorem. For any simple curve $\eta$ on the once-punctured torus, the operator $T_{r}^{\eta}$ is trigonometric, its degree is equal to the geometric intersection of $\eta$ and $\gamma$, and denoting by $\sigma^{\eta}$ its $\psi$-symbol, we have

$$
\sigma^{\gamma}=f_{\gamma}+\left(\frac{1}{2 i} \sum_{e} \frac{\partial^{2} f_{\gamma}}{\partial \theta_{e} \partial \tau_{e}}\right) \hbar+o(\hbar) .
$$

Proof. If $\eta$ corresponds to $\infty, 0$ or 1 , it is proved by the direct computations of Subsection [3.1. Given any $\eta$ parameterized by a vertex $q=[a, b]$ in the Farey triangulation, let $n$ be the number of edges crossed by the geodesic $[p, q]$ where $p$ is a point in the triangle $[0,1, \infty]$ : we call it the depth of $\eta$. We observe that the geometric intersection of $\eta$ with $\gamma$ is equal to $|b|$ : we call this quantity the degree of $\eta$. Then we show the theorem by induction on the depth. Suppose that it holds for all curves with depth less than $n$ and choose $q$ of depth $n+1$. Then the last edge crossed by the geodesic $[p, q]$ joins two curves $\gamma_{1}, \gamma_{2}$ of depth $n$. Let $\gamma_{3}$ be the symmetric of $\eta$ with respect to the geodesic $\left[\gamma_{1}, \gamma_{2}\right]$ : this vertex has depth $n-1$ and we have $\eta=A^{-1} \gamma_{1} \cdot \gamma_{2}-A^{-2} \gamma_{3}$. As the degree of $\gamma_{3}$ is strictly less than the sum of the degrees of $\gamma_{1}$ and $\gamma_{2}$, we obtain that the degree of $\eta$ is the sum of the degrees of $\gamma_{1}$ and $\gamma_{2}$. A direct application of Proposition 3.9 shows that the Theorem holds for $\eta$ of depth $n+1$ and the Theorem is proved. 
Let $\Sigma$ be a sphere with four marked points $p_{1}, p_{2}, p_{3}, p_{4}$. We can use the same argument as before using the following trick: identify $\Sigma$ with the quotient $\mathbb{R}^{2} / \mathbb{Z}^{2} \rtimes$ $\mathbb{Z}_{2}$ where $\mathbb{Z}^{2} \rtimes \mathbb{Z}_{2}$ acts on $\mathbb{R}^{2}$ by $\left(n_{1}, n_{2}, \epsilon\right) \cdot\left(x_{1}, x_{2}\right)=\left(n_{1}+\epsilon x_{1}, n_{2}+\epsilon x_{2}\right)$ and we have $p_{1}=[(0,0)], p_{2}=[(1 / 2,0)], p_{3}=[(0,1 / 2)]$ and $p_{4}=[(1 / 2,1 / 2)]$.

The double cover $T=\mathbb{R}^{2} / \mathbb{Z}^{2}$ is a ramified double cover of $\Sigma$. Any simple curve $\gamma$ in $\Sigma \backslash\left\{p_{1}, p_{2}, p_{3}, p_{4}\right\}$ lifts to a curve in $T$. We associate to it the corresponding slope in the Farey triangulation. If two curves $\gamma_{1}$ and $\gamma_{2}$ on $\Sigma$ meet twice, the corresponding vertices in the Farey triangulation form an edge. Finally, one can write in $K(\Sigma, A)$ the formula $\gamma_{1} \cdot \gamma_{2}=A^{2} \delta_{1}+A^{-2} \delta_{2}+R$ where $\delta_{1}, \delta_{2}$ are the nontrivial curves obtained by smoothing $\gamma_{1} \cup \gamma_{2}$ and $R$ is a combination of boundary curves which can be treated as constants. Hence, we deduce the following theorem:

Theorem. For any simple curve $\eta$ on the 4-times-punctured sphere, the operator $T_{r}^{\eta}$ is trigonometric, its degree is equal to half the geometric intersection of $\eta$ and $\zeta$, and denoting by $\sigma^{\eta}$ its $\psi$-symbol, we have

$$
\sigma^{\gamma}=f_{\gamma}+\left(\frac{1}{2 i} \sum_{e} \frac{\partial^{2} f_{\gamma}}{\partial \theta_{e} \partial \tau_{e}}\right) \hbar+o(\hbar)
$$

\section{Curve operators as Toeplitz operators}

In this section we will establish the fact that the matrices $T_{r}^{\gamma}$ defined in the preceding section are the matrices of Toeplitz operators on the sphere, associated to symbols with specific regularity.

\subsection{Toeplitz operators.}

4.1.1. Matrices of Toeplitz operators. In this section we will consider the quantization of the sphere in a very down-to-earth way. Given an integer $N$, we define the space $\mathcal{H}_{N}$ of polynomials in the complex variable $z$ of order strictly less than $N$ and set

$$
\langle P, Q\rangle=\frac{i}{2 \pi} \int_{\mathbb{C}} \frac{P(z) \overline{Q(z)}}{\left(1+|z|^{2}\right)^{N+1}} d z d \bar{z} \quad \text { and } \quad \varphi_{n}^{N}(z)=\sqrt{\frac{N !}{n !(N-1-n) !}} z^{n} .
$$

The vectors $\left(\varphi_{n}^{N}\right)_{n=0 \ldots N-1}$ form an orthonormal basis of $\mathcal{H}_{N}$.

By the stereographic projection

$$
S^{2} \ni(\tau, \theta) \in[0,1] \times S^{1} \rightarrow z=\sqrt{\frac{\tau}{1-\tau}} e^{i \theta} \in \mathbb{C} \cup\{\infty\} .
$$

The space $\mathcal{H}_{N}$ can be seen as a space of functions on the sphere (with a specific behavior at the north pole). Write $d \mu_{N}$ the measure $\frac{i}{2 \pi} \frac{d z d \bar{z}}{\left(1+|z|^{2}\right)^{N+1}}$. As a space of analytic functions in $L^{2}\left(\mathbb{C}, d \mu_{N}\right)$, the space $\mathcal{H}_{N}$ is closed.

For $z_{0} \in \mathbb{C}$, we define the coherent state

$$
\rho_{z_{0}}(z)=N\left(1+\bar{z}_{0} z\right)^{N-1} .
$$

These vectors satisfy $\left\langle f, \rho_{z_{0}}\right\rangle=f\left(z_{0}\right)$ for any $f \in \mathcal{H}_{N}$ and the orthogonal projector $\pi_{N}: L^{2}\left(\mathbb{C}, d \mu_{N}\right) \rightarrow \mathcal{H}_{N}$ satisfies $\left(\pi_{N} \psi\right)(z)=\left\langle\psi, \rho_{z}\right\rangle$.

For $f \in C^{\infty}\left(S^{2}, \mathbb{R}\right)$ we define an operator $T_{f}: \mathcal{H}_{N} \rightarrow \mathcal{H}_{N}$ by the equation $T_{f} \psi=$ $\pi_{N}(f \psi)$. A Toeplitz operator on $S^{2}$ is a sequence of operators $\left(T_{N}\right) \in \operatorname{End}\left(\mathcal{H}_{N}\right)$ 
such that there exists a sequence $f_{k} \in C^{\infty}\left(S^{2}, \mathbb{R}\right)$ such that for any integer $M$ the operator $R_{N}^{M}$ defined by the equation

$$
T_{N}=\sum_{k=0}^{M} N^{-k} T_{f_{k}}+R_{N}^{M}
$$

is a bounded operator whose norm satisfies $\left\|R_{M}\right\|=O\left(N^{-M-1}\right)$.

An easy use of the stationary phase lemma shows that the (anti-)Wick symbol of $T_{f}$, namely $\frac{\left\langle T_{f} \rho_{z}, \rho_{z}\right\rangle}{\left\langle\rho_{z}, \rho_{z}\right\rangle}$ satisfies

$$
\frac{\left\langle T_{f} \rho_{z}, \rho_{z}\right\rangle}{\left\langle\rho_{z}, \rho_{z}\right\rangle}=f+\frac{1}{N} \Delta_{S} f+O\left(N^{-2}\right),
$$

where $\Delta_{S}=\left(1+|z|^{2}\right)^{2} \partial_{z} \partial_{\bar{z}}$ is the Laplacian on the sphere.

4.1.2. From matrix elements to the total symbol. In this section we give an explicit formula for computing the exact Toeplitz symbol out of the matrix elements of a given operator expressed by a matrix in the basis $\varphi_{n}^{N}$ defined by (10). The key idea is the following remark.

The matrix element of an operator of the form $T_{f}: \mathcal{H}_{N} \rightarrow \mathcal{H}_{N}$ between $\varphi_{n}^{N}$ and $\varphi_{m}^{N}$ is given by the formula

$$
\begin{aligned}
F_{m, n} & =\left\langle T_{f} \varphi_{n}^{N}, \varphi_{m}^{N}\right\rangle \\
& =\frac{i}{2 \pi} \int f(z, \bar{z}) z^{n} \bar{z}^{m} \frac{d z d \bar{z}}{\left(1+|z|^{2}\right)^{N+1}} \sqrt{\frac{N !}{n !(N-1-n) !} \frac{N !}{m !(N-1-m) !}} .
\end{aligned}
$$

If we suppose that $f$ is real valued, the matrix of $T_{f}$ is Hermitian and setting $z=\sqrt{\rho} e^{i \theta}$ we can expand $f$ into Fourier series:

$$
f\left(\sqrt{\rho} e^{i \theta}, \sqrt{\rho} e^{-i \theta}\right)=\sum_{\mu \in \mathbb{Z}} f_{\mu}(\rho) e^{i \mu \theta},
$$

where $f_{-\mu}=\overline{f_{\mu}}$. Therefore we have that:

$$
\left\{\begin{array}{l}
F_{n, n+\mu}=\int_{0}^{\infty} f_{\mu}(\rho) \rho^{n+\frac{\mu}{2}} \frac{d \rho}{(1+\rho)^{N+1}} \frac{N !}{\sqrt{n !(N-1-n) !((n+\mu) !(N-1-n-\mu) !}}, \\
\overline{F_{n, n-\mu}}=\int_{0}^{\infty} f_{\mu}(\rho) \rho^{n-\frac{\mu}{2}} \frac{d \rho}{(1+\rho)^{N+1}} \frac{N !}{\sqrt{n !(N-1-n) !((n-\mu) !(N-1-n+\mu) !}} .
\end{array}\right.
$$

Let us define the Mellin transform $\mathcal{M} f$ of $f$ as

$$
\mathcal{M} f(s)=\int_{0}^{\infty} x^{s} f(x) \frac{d x}{x}
$$

and its inverse

$$
\mathcal{M}^{-1} F(x)=\frac{1}{2 \pi i} \int_{c-i \infty}^{c+i \infty} x^{-s} F(s) d s
$$

We easily get

\section{Proposition 4.1.}

$$
f_{\mu}(\rho)=\frac{1}{2 i \pi} \rho^{-1-\frac{\mu}{2}}(1+\rho)^{N+1} \int_{c-i \infty}^{c+i \infty} F_{s, s+\mu} \rho^{-s} C(N, \mu, s) d s
$$

and

$$
\overline{f_{\mu}(\rho)}=\frac{1}{2 i \pi} \rho^{-1+\frac{\mu}{2}}(1+\rho)^{N+1} \int_{c-i \infty}^{c+i \infty} F_{s, s-\mu} \rho^{-s} C(N,-\mu, s) d s
$$


for a convenient value of $c \in \mathbb{R}$ and where

$$
C(N, \mu, s)=\frac{\sqrt{\Gamma(s+1) \Gamma(s+\mu+1) \Gamma(N-s) \Gamma(N-s-\mu)}}{\Gamma(N+1)} .
$$

Write $C(N, \mu, s)=E(N, \mu, s) \Pi(N, s)$ where

$$
\Pi(N, s)=\frac{\Gamma(s+1) \Gamma(N-s)}{\Gamma(N+1)} .
$$

We compute that

$$
E(N, \mu, s)=\left\{\begin{array}{c}
\left(\prod_{k=1}^{\mu} \frac{s+k}{N-s-k}\right)^{1 / 2} \text { if } \mu>0 \\
\left(\prod_{k=1}^{-\mu} \frac{N-s-1+k}{s+1-k}\right)^{1 / 2} \text { if } \mu<0, \\
1 \text { if } \mu=0 .
\end{array}\right.
$$

Theorem 4.2. Fix $N$ and $k$ two integers with $k<N$. Let $T$ be an operator in $\mathcal{H}_{N}$ whose matrix elements are $F_{m, n}$ in the basis $\left\{\varphi_{n}^{N}\right\}_{n=0, \ldots, N-1}$ and vanish for $|m-n|>k$. Suppose that the following assumptions hold:

- For any integer $\mu$ such that $|\mu| \leq k$, there is an analytic extension of $F_{s, s+\mu} E(N, \mu, s)$ to a function $G(\mu, s)$ holomorphic in $s$ on a strip $M_{1}+$ $k / 2<\operatorname{Re}(s)+\mu / 2<M_{2}-k / 2$.

- For all $s$ and $\mu, \overline{G(-\mu, s+\mu)} E(N, \mu, \bar{s})^{2}=G(\mu, \bar{s})$.

- There are constants $\alpha, \beta$ such that $|G(\mu, s)| \leq \alpha \exp (\beta|\operatorname{Im} s|)$ for all $s$ such that $M_{1}+k / 2<\operatorname{Re}(s)+\mu / 2<M_{2}-k / 2$. Moreover $\beta$ satisfies $\beta<\pi$.

Suppose that $M_{1}<-k-1$ and $M_{2}>N+k$. Then the formulas of Proposition 4.1 define a function $f$ on the sphere such that $T=T_{f}$. Moreover $f$ is

- of class $C^{\infty}$ on the sphere minus the poles,

- of class $C^{M_{S}}$ at the south pole for any $M_{S}<-M_{1}-1-k$,

- of class $C^{M_{N}}$ at the north pole for any $M_{N}<M_{2}-N-k$.

Remark 4.3. The second hypothesis is an enhancement of the Hermitian condition $F_{\bar{s}, \bar{s}+\mu}=\overline{F_{s+\mu, s}}$, observing that for $s \in \mathbb{R}, E(N,-\mu, s+\mu)=E(N, \mu, s)^{-1}$. It follows that $G(\mu, s)$ vanishes when $E(N, \mu, s)^{2}$ does, that is, if $s+\mu \in\{0, \ldots, N-1\}$ but $s \notin\{0, \ldots, N-1\}$.

Proof. Let us start with the following remark.

Remark 4.4. In order that a function $f$ on the sphere, regular away from the poles, defines an operator through the formula $T=T_{f}$ at fixed value of $N$, we only need that all the matrix elements exist, which means that the function has to be integrable at the poles. This property is reflected by the holomorphy conditions on the functions $G(\mu, s)$ as we are going to see now.

Using many times the formula $\Gamma(s+1)=s \Gamma(s)$ and the reflection formula $\Gamma(s) \Gamma(1-s)=\frac{\pi}{\sin (\pi s)}$ we get

$$
\Pi(N, s)=\frac{\pi s(1-s)(2-s) \cdots(N-1-s)}{\sin (\pi s) N !} .
$$

If $\operatorname{Re}(s)$ remains bounded and writing $\xi=\operatorname{Im} s$, we deduce the following uniform estimate:

$$
\Pi(N, s) \underset{|\xi| \rightarrow \infty}{\sim} \frac{\pi|\xi|^{N}}{e^{\pi|\xi|} N !} .
$$


Rewriting the first formula of Proposition 4.1 we have

$$
f_{\mu}(\rho)=\frac{1}{2 i \pi} \rho^{-1-\frac{\mu}{2}}(1+\rho)^{N+1} \int_{c-i \infty}^{c+i \infty} G(\mu, s) \rho^{-s} \Pi(N, s) d s .
$$

The estimation $|G(\mu, s)| \leq \alpha \exp (\beta|s|)$ implies that the integral in the proposition is well defined and smooth for $\rho \in(0, \infty)$ as soon as $\beta<\pi$. In order to get the regularity property we need to show that

$$
\begin{aligned}
& \text { - } f_{\mu} \rho^{-\frac{\mu}{2}} \in C^{M_{S}} \text { for } \rho \sim 0 . \\
& \text { - } f_{\mu} \rho^{+\frac{\mu}{2}} \in C^{M_{N}} \text { for } \rho \sim \infty .
\end{aligned}
$$

We do the proof for $\rho \sim 0$, the case $\rho \sim \infty$ being the same.

Taking the constant $c=M_{1}+\frac{k-\mu}{2}+\epsilon$ we get by the residue formula

$$
f_{\mu}(\rho)=-\rho^{-1-\frac{\mu}{2}}(1+\rho)^{N+1} \sum_{c<\ell<0} G(\mu, l)\left(\begin{array}{c}
N-l-1 \\
-l-1
\end{array}\right) \rho^{-\ell}+O\left(\rho^{-M_{1}-1-\frac{k}{2}-\epsilon}\right) .
$$

If $\mu>0$, we have that $G(\mu, l)=0$ if $l<0$ and $l+\mu \geq 0$. Hence the first non-zero term in the sum is for $l=-1-\mu$. This shows that one can factor $\rho^{\frac{\mu}{2}}$ in the sum as expected. If $\mu<0$, the first non-zero residue is for $l=-1$, and this time, one can factor the term $\rho^{-\frac{\mu}{2}}$.

We deduce the $C^{M_{S}}$ regularity property where $M_{S}$ is the highest integer with $M_{S}<-M_{1}-1-k$.

\subsubsection{Asymptotic expansion.}

Theorem 4.5. Let $T^{N}$ be a sequence of operators in $\mathcal{H}_{N}$ whose matrix elements are denoted by $F_{n, n+\mu}=F_{n, n+\mu}^{N}$. Let $g\left(\mu, \tau, \frac{1}{N}\right)$ be a family of functions holomorphic in $\tau$ satisfying

(22) $g\left(\mu, \frac{n}{N}, \frac{1}{N}\right)=F_{n, n+\mu} E(N, \mu, n)$ for all $n$ such that $n, n+\mu \in\{0, \ldots, N-1\}$.

We suppose that

- There exists $M_{1}<0<1<M_{2}$ such that $g\left(\mu, \tau, \frac{1}{N}\right)$ are holomorphic on the strip $M_{1}+\frac{k}{2 N}<\operatorname{Re}(\tau)+\frac{\mu}{2 N}<M_{2}-\frac{k}{2 N}$ for some $k>0$ and have an asymptotic expansion when $N$ goes to infinity which is uniform on compact sets.

- $\overline{g\left(-\mu, \tau+\frac{\mu}{N}, \frac{1}{N}\right)} E(N, \mu, N \bar{\tau})^{2}=g\left(\mu, \bar{\tau}, \frac{1}{N}\right)$ for all $N$ and $\tau$.

- There are constants $\alpha, \beta$ such that one has

$$
\left|g\left(\mu, \tau, \frac{1}{N}\right)\right| \leq \alpha e^{\beta|\operatorname{Im} \tau|} \text { for } \tau \text { in the domain. }
$$

Denote by $f^{N}$ the sequence of functions given by Theorem 4.2 so that $T^{N}=T_{f^{N}}$. Then $f^{N}$ has an asymptotic expansion of the form

$$
f^{N} \sim \sum_{n=0}^{\infty} f^{(n)} N^{-n}
$$

where $f^{(n)}$ are smooth functions on $S^{2}$ and $f^{(0)}(\tau, \theta)=\sum_{\mu} f_{\mu}^{(0)}(\tau) e^{i \mu \theta}$ where $f_{\mu}^{(0)}(\tau)=\lim _{N \rightarrow \infty} F_{\lfloor N \tau\rfloor,\lfloor N \tau\rfloor+\mu}$. In other terms, the family $T^{N}$ is a family of Toeplitz operators on the sphere whose principal symbol is $f^{(0)}$. 
Proof. Suppose that $\mu \geq 0$ and consider the second formula of Proposition 4.1 .

$$
f_{-\mu}^{N}(\rho)=\frac{1}{2 i \pi} \rho^{-1+\frac{\mu}{2}}(1+\rho)^{N+1} \int_{c-i \infty}^{c+i \infty} g\left(-\mu, \frac{s}{N}, \frac{1}{N}\right) \rho^{-s} \Pi(N, s) d s .
$$

Change the variable $s=-1+\alpha$ with $\alpha=\frac{\rho}{1+\rho}+i \xi, \xi \in \mathbb{R}$.

We get

$$
f_{-\mu}^{N}(\rho)=\frac{1}{2 i \pi}(1+\rho)^{N+1} \rho^{\mu / 2} \int g\left(-\mu, \frac{-1+\alpha}{N}, \frac{1}{N}\right) \rho^{-\alpha} \Pi(N,-1+\alpha) d \alpha .
$$

Let us define $\chi_{M} \in C^{\infty}(\mathbb{R})$ such that $\chi_{M}(\xi)=1$ for $|\xi|<M$ and $\chi_{M}(\xi)=0$ for $|\xi|>M+1$. Performing a decomposition of the identity

$$
1=\chi_{M}(\xi / N)+\left(1-\chi_{M}(\xi / N)\right),
$$

we write

$$
f_{-\mu}^{N}(\rho)=\frac{1}{2 i \pi}(1+\rho)^{N+1} \rho^{\mu / 2}(J(N, \rho)+I(N, \rho)) .
$$

We first consider the integral

$I(N, \rho)=\rho^{-\frac{\rho}{1+\rho}} \int g\left(-\mu, \frac{-1+\frac{\rho}{1+\rho}+i \xi}{N}, \frac{1}{N}\right) \rho^{-i \xi} \Pi\left(N,-1+\frac{\rho}{1+\rho}+i \xi\right)\left(1-\chi_{M}\left(\frac{\xi}{N}\right)\right) d \xi$.

We show that $\left|(1+\rho)^{N+1} I(N, \rho)\right|=O\left(N^{-\infty}\right)$ uniformly for $\rho$ in compact subsets of $[0, \infty)$. Indeed we have

$$
|I(N, \rho)| \leq \int_{|\xi|>M N} C e^{\frac{\beta}{N}|\xi|}\left|\Pi\left(N,-1+\frac{\rho}{1+\rho}+i \xi\right)\right| d \xi \leq C_{1} \int_{|\xi|>M N} e^{\frac{\beta}{N}|\xi|} \frac{|\xi|^{N}}{e^{\pi|\xi|} N !} d \xi .
$$

Here we used the estimate for $\Pi(N,-1+i \xi)$ given in (20). We compute explicitly

$$
\int_{|\xi|>M N} e^{\frac{\beta}{N}|\xi|} \frac{|\xi|^{N}}{e^{\pi|\xi|} N !}=\frac{2}{\left(\pi-\frac{\beta}{N}\right)^{N+1}} \frac{1}{N !} \int_{M(N \pi-\beta)}^{\infty} u^{N} e^{-u} d u .
$$

Let $N$ be big enough such that $M(N \pi-\beta) \geq(M+1) N$ and $\pi-\frac{\beta}{N} \geq 1$. We get $|I(N, \rho)| \leq C_{2}(M+1)^{N} e^{-M N}$ as an application of the following elementary lemma:

Lemma 4.6. For $a \geq 1$, we have $\frac{1}{N !} \int_{a N}^{\infty} u^{N} e^{-u} d u \leq a^{N} e^{(1-a) N}$.

This shows that $(1+\rho)^{N+1} I(N, \rho)$ is $O\left(N^{-\infty}\right)$ uniformly for $\rho \leq \frac{e^{M}}{2 M}$ and hence on any compact sets as $M$ can be chosen arbitrarily big.

For $J(N, \rho)$, remarking that $\Pi(N, s)=\frac{\Gamma(s+1) \Gamma(N-s)}{\Gamma(N+1)}=\int_{0}^{\infty} \frac{u^{s} d u}{(1+u)^{N+1}}$ if $-1<$ Re $s<N$ and multiplying $\alpha$ by $N$, we get

$$
J(N, \rho)=N \int g\left(-\mu,-1 / N+\alpha, \frac{1}{N}\right) \rho^{-N \alpha} \int_{0}^{\infty} \frac{u^{-1+N \alpha} d u}{(1+u)^{N+1}} \chi(\operatorname{Im} \alpha) d \alpha .
$$

Putting $u=\rho v$ in the second integral and using the Fubini Theorem we get

$$
\begin{aligned}
J(N, \rho) & =N \int d \alpha \int_{0}^{\infty} d v g\left(-\mu,-1 / N+\alpha, \frac{1}{N}\right) \chi(\operatorname{Im} \alpha) \frac{v^{-1+N \alpha}}{(1+\rho v)^{N+1}} \\
& =N \int d \alpha \int_{0}^{\infty} d v g\left(-\mu,-1 / N+\alpha, \frac{1}{N}\right) \chi(\operatorname{Im} \alpha) \frac{e^{N(\alpha \ln v-\ln (1+\rho v))}}{v(1+\rho v)} .
\end{aligned}
$$

The phase $\Phi(\alpha, v)=\alpha \ln v-\ln (1+\rho v)$ is stationary for $v=1$ and $\alpha=\frac{\rho}{1+\rho}$, that is, $v=1, \xi=0$. Moreover the determinant of the Hessian at the stationary point is equal to 1 ; therefore, we can apply the stationary phase lemma uniformly 
up to $\rho \rightarrow 0$ for the part on the integral near the stationary point. For the rest of the integral we notice that $\Phi\left(\frac{\rho}{1+\rho}+i \xi, v\right)=-\log (1+\rho)+\Phi_{1}(\xi, v)$ with $\operatorname{Re} \Phi_{1}<0$ for $|\xi|>0$. Therefore the non-stationary phase lemma gives that the contribution to $J(N, \rho)$ given by $|\xi|>0$ in the integral (23) is $O\left((1+\rho)^{-N} N^{-\infty}\right)$.

We get

$$
J(N, \rho) \sim \frac{2 i \pi}{N}(1+\rho)^{-(N+1)} \sum_{k=0}^{\infty} N^{-k} g_{k}\left(-\mu, \frac{\rho}{1+\rho}\right)
$$

where $g_{0}=g\left(-\mu, \frac{\rho}{1+\rho}, 0\right)$ and $g_{k}(-\mu, \tau)$ are smooth functions of $\tau \in[0,+\infty)$.

Putting everything together we get

$$
f_{-\mu}^{N}(\rho) \sim \rho^{\mu / 2} \sum_{k=0}^{\infty} N^{-k} g_{k}\left(-\mu, \frac{\rho}{1+\rho}\right) .
$$

This proves that for any $k$, the function $f_{k}(\tau, \theta)=\sum_{\mu} \rho^{-\mu / 2} g_{k}(-\mu, \tau) e^{i \mu \theta}$ is a smooth function at the south pole.

From E (22) we get $G\left(-\mu, \tau, \frac{1}{N}\right)=F_{N \tau, N \tau-\mu} E(N,-\mu, N \tau)$ and from (18) we have $E(N,-\mu, N \tau)=\left(\frac{1-\tau}{\tau}\right)^{\mu / 2}=\rho^{-\mu / 2}$. Hence, $f_{\mu}^{N}(\rho) \simeq f_{\mu}^{(0)}(\tau)$ as claimed in the theorem.

4.2. Curve operators as Toeplitz operators. Let us relate Theorem 4.2 to TQFT and curve operators. Let $\Sigma$ be either the once-punctured torus or the 4times-punctured sphere. Let $r$ be an integer and $\check{c}$ be an admissible coloring of the marked points of $\Sigma$. We consider the following two cases:

(1) If $\Sigma$ is a torus, let $r$ be the level and $a$ the (odd) color of the marked point. The basis vectors of $V_{r}(\Sigma, \check{c})$ are parametrized by an integer $m \in$ $(a / 2, r-a / 2)$. Hence its dimension is $N=r-a$. We identify the basis $\varphi_{m}$ of $V_{r}(\Sigma, a)$ and the basis $\varphi_{n}^{N}$ of $\mathcal{H}_{N}$ by setting $m=n+\frac{a+1}{2}$ for $0 \leq n<N$.

(2) If $\Sigma$ is a sphere, we write $\check{c}=(a, b, c, d)$. The basis vectors of $V_{r}(\Sigma, \check{c})$ are parametrized by an integer $m$ satisfying $\max (|a-d|,|b-c|)<m<$ $\min (a+d, 2 r-a-d, b+c, 2 r-b-c)$ and $m+1=a+d=b+c \bmod 2$. Its dimension is $N$ where

$$
N=\frac{1}{2}(\min (a+d, 2 r-a-d, b+c, 2 r-b-c)-\max (|a-d|,|b-c|)) .
$$

We identify the basis $\varphi_{m}$ of $V_{r}(\Sigma, \check{c})$ and $\varphi_{n}^{N}$ of $\mathcal{H}_{N}$ by setting $m=$ $\max (|a-d|,|b-c|)+1+2 n$ for $0 \leq n<N$.

In any case, we define an isomorphism $I_{r}: V_{r}(\Sigma, \check{c}) \simeq \mathcal{H}_{N}$. Fix a curve $\gamma$ on $\Sigma$. The curve operator $T_{r}^{\gamma}$ gives a matrix $I_{r} T_{r}^{\gamma} I_{r}^{-1} \in \operatorname{End}\left(\mathcal{H}_{N}\right)$ that we also denote by $T_{r}^{\gamma}$ for short. We first prove in Subsection 4.2.1 the following theorem:

Theorem 4.7. Let $\Sigma$ be either the once-punctured torus or the 4-times-punctured sphere, $r$ a level and $\check{c}$ an admissible coloring of the marked points. Let $N=$ $\operatorname{dim} V_{r}(\Sigma, \check{c})$ and let $I_{r}: V_{r}(\Sigma, \check{c}) \simeq \mathcal{H}_{N}$ be the isomorphism described above.

Let $\gamma$ be a curve on $\Sigma$ of degree $k$. Suppose that the quantity $M_{P}=\frac{a}{2}-1-k$ is non-negative in the torus case and that the quantities $M_{S}=M-1-k$ and $M_{N}=r-m-k$ are non-negative in the sphere case where $M=\frac{1}{2} \max (|a-d|,|c-d|)$ and $m=\frac{1}{2} \min (a+d, 2 r-a-d, b+c, 2 r-b-c)$.

Then, there is a function $f_{r}^{\gamma}$ on the sphere such that

$$
T_{r}^{\gamma}=T_{f_{r}^{\gamma}} .
$$


This function $f_{r}^{\gamma}$ is smooth away from the poles and is

- of class $\left\lfloor M_{P}\right\rfloor$ at the poles in the torus case,

- of class $\left\lfloor M_{S}\right\rfloor$ at the south pole and of class $\left\lfloor M_{N}\right\rfloor$ at the north pole in the case of the 4-times-punctured sphere.

4.2.1. Analytic continuation of matrix elements. Let $\gamma$ be a curve of degree $k$ in $\Sigma$. Theorem 2.5 states that there exists coefficients $F_{n, n+\mu}$ for $|\mu| \leq k$ such that $T_{r}^{\gamma} \varphi_{n}=\sum_{\mu} F_{n, n+\mu} \varphi_{n+\mu}$.

The formulas for $F_{n, n+\mu}$ are rational expressions involving square roots, trigonometric functions, the parameter $n$, the coloring $\check{c}$ and the level $r$. There is an obvious extension of $F_{n, n+\mu}$ to a multivalued analytic function $F_{s, s+\mu}$ whose domain contains $[0, N-1] \cap([0, N-1]-\mu)$.

Definition 4.8. For any curve $\gamma$, set

$$
G^{\gamma}(\mu, s)=F_{s, s+\mu} E(N, \mu, s) .
$$

We will also use the following definition in order to estimate $G(\mu, s)$.

Definition 4.9. Let $f(s)$ be some meromorphic function in a domain of the form $x<\operatorname{Re}(s)<y$. Given some $\beta \geq 0$ we will say that $f$ has order $\beta$ if there exists $M, \alpha, \alpha^{\prime}>0$ such that for any $s$ with $|\operatorname{Im}(s)| \geq M$ and $x<\operatorname{Re}(s)<y$ we have

$$
\alpha e^{\beta|\operatorname{Im}(s)|} \leq|f(s)| \leq \alpha^{\prime} e^{\beta|\operatorname{Im}(s)|} .
$$

Proposition 4.10. Let $\Sigma$ be either a once-punctured torus or a 4-times-punctured sphere. Let $\check{c}$ be an admissible coloring of level $r$. Let $N$ be the dimension of $V_{r}(\Sigma, \check{c})$ and consider the isometric spaces $V_{r}(\Sigma, \check{c}) \simeq \mathcal{H}_{N}$.

For any curve $\gamma \subset \Sigma$ of degree $k$, let $G^{\gamma}(\mu, s)$ be the analytic functions associated to $T_{r}^{\gamma}$ by Definition 4.8. Then

(1) $G^{\gamma}(\mu, s)$ is holomorphic for $M_{1}+k / 2<\operatorname{Re}(s)+\mu / 2<M_{2}-k / 2$.

(2) There exists $\beta$ depending only on $\gamma$ such that for all $\mu, G(\mu, s)$ have order $\frac{\beta}{r}$.

Where $M_{1}=-a / 2$ and $M_{2}=N-1+a / 2$ in the torus case. In the sphere case, $M_{1}=-M$ and $M_{2}=N-1+r-M$ where $M=\frac{1}{2} \max (|a-d|,|b-c|)$.

Proof. The proof consists of checking it for the generators and the use of Kauffman relations. The second property is obvious in all the explicit formulas so we will not discuss it.

\section{Torus case}

Let $\gamma$ be the curve of Figure [6. Then $\mu=0$ and $E(N, 0, s)=1$. The two statements are obvious from the following expression:

$$
G_{N}^{\gamma}(0, s)=-2 \cos \left(\frac{\pi}{2 r}(2 s+a+1)\right) .
$$

The curve $\delta$ satisfies

$$
G_{N}^{\delta}(1, s)=-\sqrt{\frac{\sin \left(\frac{\pi}{r}(s+a+1)\right) \sin \left(\frac{\pi}{r}(s+1)\right)}{\sin \left(\frac{\pi}{2 r}(2 s+a+1)\right) \sin \left(\frac{\pi}{2 r}(2 s+a+3)\right)}} E(N, 1, s) .
$$

The square of the first factor has poles at $s=-(a+1) / 2$ and $s=-(a+3) / 2$ modulo $r$ and zeroes at $s=-1$ and $s=r-a-1$ modulo $r$. On the other hand, one has $E(N, 1, s)=\sqrt{\frac{s+1}{N-s-1}}$. It follows that $G^{\delta}(1, s)$ is holomorphic for $-\frac{a+1}{2}<s<r-\frac{a+3}{2}$ as expected. 
The same kind of computation shows that $G_{N}^{\delta}(-1, s)$ is holomorphic for $-\frac{a-1}{2}<$ $s<r-\frac{a+1}{2}$. Finally, the formula

$$
G_{N}^{\xi}( \pm 1, s)=G_{N}^{\delta}( \pm 1, s) \exp \left(\frac{i \pi}{2 r}( \pm 2 s+a+2)\right)
$$

shows that the analytic properties of $G^{\delta}$ and $G^{\xi}$ are the same.

We observe that we can set $M_{1}=-a / 2$ and $M_{2}=r-a / 2-1=N-1+a / 2$. It is clear that all the $G$ functions involved here have order at most $\frac{\pi}{2 r}$.

\section{Sphere case}

It is very similar to the torus case so that we do not give more details. The general case follows from the next lemma:

Lemma 4.11. Let $T^{1}, T^{2}$ be two operators in $\mathcal{H}_{N}$ of respective degrees $k_{1}, k_{2}$ whose coefficients can be analytically continued, that is, there is a family $G^{i}(\mu, s)$ for $i=1,2$ and $|\mu| \leq k_{i}$ such that (23) is satisfied and the $G^{i}(\mu, s)$ satisfy the properties of Proposition 4.10 .

Then, the product $T^{2} T^{1}$ has the same property, meaning that its coefficients can be analytically continued via functions $G^{12}(\mu, s)$ satisfying the properties of Proposition 4.10 with $k=k_{1}+k_{2}$.

Proof. From the matrix multiplication we get for any $n \in \mathbb{Z}$ the following formula:

$$
F_{n, n+\mu}^{12}=\sum_{\nu} F_{n, n+\nu}^{1} F_{n+\nu, n+\mu}^{2}
$$

Using the identity $E(N, \mu, s)=E(N, \nu, s) E(N, \mu-\nu, s+\nu)$ we get:

$$
G^{12}(\mu, s)=\sum_{\nu} G^{1}(\nu, s) G^{2}(\mu-\nu, s+\nu) .
$$

Take $\mu$ with $|\mu| \leq k$ and consider one term of the sum with $|\nu| \leq k_{1}$ and $|\mu-\nu| \leq k_{2}$.

The factor $G^{1}(\nu, s)$ is holomorphic if $M_{1}+\frac{k_{1}}{2}<\operatorname{Re}(s)+\nu / 2<M_{2}-\frac{k_{1}}{2}$, while the second factor is holomorphic if $M_{1}+\frac{k_{2}}{2}<\operatorname{Re}\left(s+\frac{\nu}{2}\right)+\frac{\mu-\nu}{2}<M_{2}-\frac{k_{2}}{2}$. We deduce that if $M_{1}+\frac{k}{2}<\operatorname{Re}(s)+\frac{\mu}{2}<M_{2}-\frac{k}{2}$, the product is holomorphic, proving the first point.

It is obvious from (24) that if $G(\nu, s)$ has order $\frac{\beta_{1}}{r}$ and $G^{2}(\nu, s)$ has order $\frac{\beta_{2}}{r}$ for all $\mu$ and $\nu$, then $G(\mu, s)$ has order $\frac{\beta_{1}+\beta_{2}}{r}$. This proves the last point.

The proof of Proposition 4.10 follows from the particular cases and the product formula by an induction very similar to the one in the proof of Theorem 2.5.

4.2.2. Asymptotic regime. Let $\Sigma$ be either the punctured torus or the 4-timespunctured sphere. Let $D$ be an odd level and $\check{c}$ be an admissible coloring of the marked points of $\Sigma$. For any odd integer $\bar{r}$, set $r=D \bar{r}$. The coloring $\bar{r} \check{c}$ is admissible, hence the family of vector spaces $V_{r}(\Sigma, \bar{r} \check{c})$ is well defined and its dimension grows linearly. To be more precise, consider the two cases we focus on:

(1) If $\Sigma$ is a torus, let $D$ be the level and $a$ the color of the marked point. The space $V_{r}(\Sigma, a \bar{r})$ has dimension $\bar{r} \Delta$ where $\Delta=D-a$.

(2) If $\Sigma$ is a sphere, we write $\check{c}=(a, b, c, d)$. The space $V_{r}(\Sigma, \bar{r} \check{c})$ has dimension $\bar{r} \Delta$ where

$$
\Delta=\frac{1}{2}(\min (a+d, 2 D-a-d, b+c, 2 D-b-c)-\max (|a-d|,|b-c|)) .
$$


In any case, we define a sequence of isomorphisms $I_{r}: V_{r}(\Sigma, \bar{r} \check{c}) \simeq \mathcal{H}_{\bar{r} \Delta}$ by identifying the canonical Hermitian basis of both spaces.

Theorem 4.12. Let $\Sigma$ be either the once-punctured torus or the 4-times-punctured surface and $\gamma$ be a curve in $\Sigma$. By Proposition 4.10, for any $r=\bar{r} D$, let $f_{r}^{\gamma}$ be the function on the standard sphere such that $T_{r}^{\gamma}=T_{f_{r}^{\gamma}}$.

Suppose that in the sphere case one has either $a \neq d$ or $b \neq c$. Then the sequence $f_{r}^{\gamma}$ is an asymptotic expansion

$$
f_{r}^{\gamma}=\sum_{n \geq 0}^{\infty} r^{-n} f_{(n)}^{\gamma}
$$

where the functions $f_{(n)}^{\gamma}$ are $C^{\infty}$ on the sphere and $f_{(0)}^{\gamma}=f_{\gamma}$. In other words, the family $T_{r}^{\gamma}$ is a Toeplitz operator with principal symbol $f_{\gamma}$.

This is an application of Theorem4.5. The only hypothesis which was not proved in Proposition 4.10 is an analytic version of Theorem 2.5 which is stated below.

Moreover the proof of Theorem 4.5 permits us to compute, by using subleading order in the stationary phase lemma, the sub-principal symbol of the curve operators. However, we will give in Subsection 5.3 a less tedious way of getting it.

Remark 4.13. It is important in the hypothesis of Theorem 4.12 that one has $M_{1}<0<N-1<M_{2}$. This will ensure that for any $\gamma$ the exact symbols $f_{r}^{\gamma}$ will have increasing regularity at the poles when $r$ is large. This property is provided by the hypothesis in Theorem 4.12. No hypothesis is needed in the torus case as the condition $a>0$ is automatic from the oddness of $a$.

We can relate these conditions to the singularities of the moduli spaces. In the torus case, the moduli space $\mathcal{M}\left(\Sigma, \frac{\pi a}{D}\right)$ is singular only if $a=0$ (we suppose that $\Delta=D-a>0$ and hence $a \neq D)$. In the sphere case, it is well known that the moduli space $\mathcal{M}\left(\Sigma, \frac{\pi a}{D}, \frac{\pi b}{D}, \frac{\pi c}{D}, \frac{\pi d}{D}\right)$ is singular if and only if one has $a \pm b \pm c \pm d \neq 0$ $\bmod 2 D$ for all choices of signs. In particular, Theorem 4.12 applies in all regular cases.

Remark 4.14. We remark that we could have checked that the theorem holds for the generators and invoke the well-known but non-trivial fact that a product of Toeplitz operators is a Toeplitz operator.

Proposition 4.15. Let $\Sigma$ be either a once-punctured torus or a 4-times-punctured sphere. Let $\check{c}$ be an admissible coloring of level D. Let $\bar{r}$ be an odd integer and write $r=D \bar{r}$ and $N=\Delta \bar{r}$. Let $M_{1}$ and $M_{2}$ be defined as in Proposition 4.10. We suppose that $M_{1}<0$ and $M_{2}>N-1$.

For any curve $\gamma \subset \Sigma$ of degree $k$, write $g^{\gamma}\left(\mu, \tau, \frac{1}{N}\right)=F_{s, s+\mu}^{N} E(N, \mu, N \tau)$ where $F_{n, m}^{N}$ are the matrix elements of $T_{r}^{\gamma}, \tau=\frac{s}{N}$. Then, $g^{\gamma}\left(\mu, \tau, \frac{1}{N}\right)$ is an analytic function for $M_{1}+\frac{k}{2 N}<\operatorname{Re}(\tau)+\frac{\mu}{2 N}<M_{2}-\frac{k}{2 N}$. On any compact subsets of the strip $M_{1}<\operatorname{Re}(\tau)<M_{2}$, one has the following expansion:

$$
g^{\gamma}(\mu, \tau, \hbar)=\sum_{n=0}^{\infty} N^{-n} g_{(n)}^{\gamma}(\mu, \tau) .
$$

Moreover, one has, for $f_{\gamma}$ defined in Theorem 2.2 ,

$$
f_{\gamma}=\sum_{\mu}\left(\frac{1-\tau}{\tau}\right)^{\mu / 2} g_{(0)}^{\gamma}(\mu, \tau) e^{i \mu \theta} .
$$


Proof. The first result is obvious on the generators of the Kauffman algebra and is stable by taking products. The second statement is a direct consequence of Theorem 2.5 .

\section{Asymptotics of MAPPING GROUP REPRESENTATIONS}

The aim of this section is to present some applications of the Toeplitz calculus introduced in the preceding section to the computation of the asymptotics of the coefficients of the quantum representations of the mapping class group. In particular we want to recover in a systematic way the results of [TW05] on the asymptotics of the $6 \mathrm{j}$-symbols.

5.1. General pairing. In this section we compute the leading order of the scalar product between any two eigenvectors of any two curve-operators in the limit $N \rightarrow$ $\infty$ under the condition that the intersection of the underlying Bohr-Sommerfeld curves are transversal.

5.1.1. WKB quasi-modes. Since the curve operators are Toeplitz operators on the sphere, it is well known that each eigenvector can be approximated by WKB type quasi-modes, analytic in a neighborhood of the underlying Bohr-Sommerfeld level set; see $[\mathrm{PU}, \mathrm{V}]$. We want to present here a computation valid microlocally away from the singular points of the symbol, and better adapted to the algebraic properties of the TQFT than the usual construction. The computation will be valid far away from the poles of the sphere, but since it is known that the construction is analytic in a neighborhood of the BS curve, the formula will be valid all around the trajectory. This method will just happen to be more efficient for the algebra of the computation. Moreover it will emphasize the role of the matrix elements of the curve operator, and will be possibly generalizable to higher genus situations.

Let $\Sigma$ be either the punctured torus or the 4-times-punctured sphere and $\check{c}$ be a coloring of the marked points with level $D$. Given any odd number $\bar{r}$, we fix $N=\Delta \bar{r}$ and $r=D \bar{r}$ and consider the isomorphic spaces $V_{r}(\Sigma, \bar{r} \check{c}) \simeq \mathcal{H}_{N}$ as in Subsection 4.2.2. We fix $\hbar=\frac{1}{N}$ and $\varphi_{n}^{N}(z)=\sqrt{\frac{N !}{n !(N-1-n) !}} z^{n}$ as previously.

Given a curve $\gamma \subset \Sigma$ of degree $k$, we denote by $T_{r}^{\gamma}$ the curve operator acting on $\mathcal{H}_{N}$ and we denote by $F_{\mu}(\tau, \hbar)$ the functions satisfying

$$
T_{r}^{\gamma} \varphi_{n}^{N}=\sum_{|\mu| \leq k} F_{\mu}(n \hbar, \hbar) \varphi_{n+\mu}^{N}
$$

Define the following operators:

$$
H \varphi_{n}^{N}=\hbar n \varphi_{n}^{N}, T^{+} \varphi_{n}^{N}=\left(1-\delta_{n, N-1}\right) \varphi_{n+1}^{N} \text { and } T^{-} \varphi_{n}^{N}=\left(1-\delta_{n, 0}\right) \varphi_{n-1}^{N} .
$$

Therefore $T_{r}^{\gamma}=\sum_{|\mu| \leq k}\left(T^{\operatorname{Sign}(\mu)}\right)^{|\mu|} F_{\mu}(H, \hbar)$. We have $H=\frac{1}{N} z \frac{d}{d z}$. Pick $\epsilon>0$ and consider the space $\mathcal{H}_{N}^{\epsilon}=\operatorname{span}\left\{\varphi_{n}, \epsilon N<n<(1-\epsilon) N\right\}$.

We first remark that, on $\mathcal{H}_{N}^{\epsilon}$,

$$
T^{ \pm}=\left(\sqrt{\frac{1-H}{H} z}\right)^{ \pm 1} .
$$

Since (27) is valid only on $\mathcal{H}_{N}^{\epsilon}$, we are going to perform the following computations in restriction to $\mathcal{H}_{N}^{\epsilon}$. In other words, we restrict microlocally the phase space far 
away from the poles of the sphere. Under this condition we can replace $T^{ \pm}$by $\left(\sqrt{\frac{1-H}{H} z}\right)^{ \pm}$.

We can write on $\mathcal{H}_{N}^{\epsilon}$,

$$
T_{r}^{\gamma}=\sum_{|\mu| \leq k}\left(\sqrt{\frac{1-H}{H} z}\right)^{\mu} F_{\mu}(H, \hbar) .
$$

Since

$$
H z=z(H+\hbar)
$$

we have, again on $\mathcal{H}_{N}^{\epsilon}$,

$$
\sqrt{\frac{1-H}{H}} z=z \sqrt{\frac{1-(H+\hbar)}{H+\hbar}}=z \sqrt{\frac{1-H}{H}}\left(1-\frac{\hbar}{2} \frac{1}{H(1-H)}\right)+O\left(\hbar^{2}\right) .
$$

We easily prove the following lemma.

Lemma 5.1. We have the following uniform estimate on $\mathcal{H}_{N}^{\epsilon}$ :

$$
\left(\sqrt{\frac{1-H}{H}} z\right)^{\mu}=z^{\mu}\left(\sqrt{\frac{1-H}{H}}\right)^{\mu}\left(1-\hbar \frac{\mu(\mu+1)}{4 H(1-H)}+O_{\mathcal{B}\left(\mathcal{H}_{N}^{\epsilon} \rightarrow \mathcal{H}_{N}\right)}\left(\hbar^{2}\right)\right) .
$$

Let us define $f(z, x, \hbar)=\sum_{\mu} z^{\mu} G_{\mu}(x, \hbar)$ where, for $\epsilon<x<1-\epsilon$,

$$
G_{\mu}(x, \hbar)=\left(\frac{1-x}{x}\right)^{\mu / 2} F_{\mu}(x, \hbar)\left(1-\hbar \frac{\mu(\mu+1)}{4 x(1-x)}+O\left(\hbar^{2}\right)\right) .
$$

We obviously have on $\mathcal{H}_{N}^{\epsilon}$ that

$$
T_{r}^{\gamma}=f(z, H, \hbar)+O_{\mathcal{B}\left(\mathcal{H}_{N}^{\epsilon} \rightarrow \mathcal{H}_{N}\right)}\left(\hbar^{2}\right) .
$$

The following is standard:

Lemma 5.2. Let $T=\sum_{\mu} z^{\mu} G_{\mu}(H, \hbar)$. Let $W(z)=W_{0}(z)+\hbar W_{\hbar}(z)$ such that

$$
f_{0}\left(z, z W_{0}^{\prime}(z)\right):=f\left(z, z W_{0}^{\prime}(z), 0\right)=E \text {. }
$$

Then

(33) $T e^{\frac{W(z)}{\hbar}}=\left(f_{0}\left(z, z W_{0}^{\prime}(z)\right)+\frac{\hbar}{2} \partial_{x}^{2} f_{0}\left(z, z W_{0}^{\prime}(z)\right)\left(z \frac{d}{d z}\right)^{2} W(z)+O\left(\hbar^{2}\right)\right) e^{\frac{W(z)}{\hbar}}$.

Proof. Once again (33) is standard in Toeplitz quantization and is essentially "algebraic". Nevertheless we give a direct proof. Remember that the ordering is chosen by $T=\sum_{\mu} z^{\mu} G_{\mu}(H, \hbar)$. Therefore we have that

$$
T=\int \hat{f}(z, t) e^{i t H} d t \chi_{[0,1]}(H)
$$

where $\hat{f}$ is the Fourier transform in the second variable of a Schwartz function on the real line equal to $f(z, x)$ on $0 \leq x \leq 1$, so that $\hat{f}$ is in the Schwartz class. We remark now that, since $H=\hbar z \frac{d}{d z}, e^{i t H} \psi(z)=\psi\left(e^{i t \hbar} z\right)=\psi(z)+i t \hbar z \psi^{\prime}(z)-$ $\frac{t^{2} \hbar^{2}}{2}\left(z^{2} \psi^{\prime \prime}(z)+z \psi^{\prime}(z)\right)+o\left(\hbar^{2}\right)$ from which we deduce the formula (33). 
It is well known by semi-classical Toeplitz theory (see e.g. [PU, $[\mathrm{V}$ ) that, for the regular part of the spectrum of a Toeplitz operator, the eigenvectors are, in a neighborhood of the energy shell, close to the WKB expression. Let us recall the argument: one can construct a WKB expression which is analytic and single determined in such a neighborhood. After projection by the Toeplitz projector, this gives rise to a vector in the Hilbert space, close to the WKB expression in the neighborhood. Using Lemma 5.2 we can compute the WKB quasi-mode up to $\hbar^{2}$, $e^{\frac{W(z)}{\hbar}}$, the following way.

Let $f(z, x, \hbar)=f_{0}(z, x)+h f_{1}(z, x)+O\left(h^{2}\right)$ and $W=W_{0}+\hbar W_{1}$ with

$$
f_{0}\left(z, z W_{0}^{\prime}(z)\right)=E,
$$

Thanks to formula (33) we compute the first order correction:

$$
\begin{aligned}
\partial_{x} f_{0}\left(z, z W_{0}^{\prime}(z)\right) z & W_{1}^{\prime}(z)+f_{1}\left(z, z W_{0}^{\prime}(z)\right)=-\frac{1}{2} \partial_{x}^{2} f_{0}\left(z, z W_{0}^{\prime}(z)\right)\left(z \frac{d}{d z}\right)^{2} W_{0}(z) \\
& =-\frac{1}{2}\left(z \frac{d}{d z}\right)\left(\partial_{x} f_{0}\left(z, z W_{0}^{\prime}(z)\right)\right)+\frac{1}{2} z \partial_{z} \partial_{x} f_{0}\left(z, z W_{0}^{\prime}(z)\right) .
\end{aligned}
$$

Therefore:

$$
W_{1}(z)=-\frac{1}{2} \log \left(\partial_{x} f_{0}\left(z, z W_{0}^{\prime}(z)\right)\right)+\int \varphi(z) d z
$$

where $\varphi(z)=\frac{\frac{1}{2} z \partial_{z} \partial_{x} f_{0}\left(z, z W_{0}^{\prime}(z)\right)-f_{1}\left(z, z W_{0}^{\prime}(z)\right)}{z \partial_{x} f_{0}\left(z, z W_{0}^{\prime}(z)\right)}$. According to Theorem 2.5, we have:

$$
F_{\mu}(x, \hbar)=F_{\mu}(x, 0)+\hbar \frac{\mu+1}{2} \partial_{x} F(x, 0)+O\left(h^{2}\right) .
$$

Notice that $\mu$ has changed to $\mu+1$ because the change between the variable $\tau=\frac{\pi m}{r}$ and the variable $x=\frac{\pi n}{r}$ has the form $\tau=\alpha+\beta\left(x+\frac{\hbar}{2}\right)$ for some constants $\alpha$ and $\beta$. The shift by $\frac{\hbar}{2}$ is the important Maslov correction.

We compute $f_{0}(z, x)=\sum_{\mu} z^{\mu}\left(\frac{1-x}{x}\right)^{\mu / 2} F_{\mu}(x, 0)$ and using formula (35) we get:

$$
f_{1}(z, x)=\sum_{\mu} z^{\mu}\left(\frac{1-x}{x}\right)^{\mu / 2}\left(\frac{\mu+1}{2} \partial_{x} F(x, 0)-\frac{\mu(\mu+1)}{4 x(1-x)} F_{\mu}(x, 0)\right) .
$$

Equation (36) implies that $\frac{1}{2} z \partial_{z} \partial_{x} f_{0}(z, x)-f_{1}(z, x)=-\frac{1}{2} \partial_{x} f_{0}$ and hence $\varphi(z)=$ $-\frac{1}{2 z}$. Hence we obtain:

$$
e^{\frac{W(z)}{h}}=\frac{1}{\sqrt{z \partial_{x} f_{0}\left(z, z W_{0}^{\prime}(z)\right)}} e^{\frac{W_{0}(z)}{h}} .
$$

Let $H(z, \bar{z})=f_{0}\left(z, \frac{|z|^{2}}{1+|z|^{2}}\right)$. This is the classical Hamiltonian in the sense that $H(z, \bar{z})=\sum_{\mu} F_{\mu}(\tau, 0) e^{i \mu \theta}$ where $(\tau, \theta)$ are the spherical coordinates associated to $(z, \bar{z})$.

Note that, although the computation has been done away from the two poles, we know that the quasi-mode is holomorphic and single-valued in a neighborhood of the energy shell. Therefore the formula (37) is also valid near the two poles.

We have $\partial_{\bar{z}} H=\frac{z}{\left(1+|z|^{2}\right)^{2}} \partial_{x} f_{0}$, hence we have proven the following:

Proposition 5.3. Let $W_{0}$ be a holomorphic solution of the Hamilton-Jacobi equation $f_{0}\left(z, z W_{0}^{\prime}(z)\right)=E$ with $E$ a regular value of the function $f_{0}\left(z, \frac{|z|^{2}}{1+|z|^{2}}\right)$ satisfying 
the Bohr-Sommerfeld condition. Then

$$
\psi_{\hbar}(z)=\frac{\left(1-z W_{0}^{\prime}\right)}{\sqrt{\partial_{\bar{z}} H\left(z, \frac{W_{0}^{\prime}}{1-z W_{0}^{\prime}}\right)}} e^{\frac{W_{0}(z)}{\hbar}}
$$

is a quasi-mode modulo $\hbar^{2}$ of $T_{r}^{\gamma}$ where $f$ and $T_{r}^{\gamma}$ are related by (31).

For any $z$ such that $H(z, \bar{z})=E$ we have

$$
\psi_{\hbar}(z)=\frac{1}{1+|z|^{2}} \frac{e^{W_{0} / \hbar}}{\sqrt{\partial_{\bar{z}} H(z, \bar{z})}} .
$$

Moreover we have

$$
d\left(W_{0}(z)-\frac{1}{2} \log \left(1+|z|^{2}\right)\right)=\frac{1}{2} \frac{\bar{z} d z-z d \bar{z}}{1+|z|^{2}}=2 i \pi \eta
$$

where $d \eta=\omega$ is the symplectic form. Let $z_{0}$ be a point satisfying $H\left(z_{0}, \overline{z_{0}}\right)=E$. We will say that the quasi-mode $\psi$ is normalized at $z_{0}$ if $W_{0}\left(z_{0}\right)=\frac{1}{2} \log \left(1+\left|z_{0}\right|^{2}\right)$. By (39), this implies that $W_{0}-\frac{1}{2} \log \left(1+|z|^{2}\right)$ is purely imaginary on the level set.

We derive easily, using the stationary phase lemma, the following lemma.

Lemma 5.4. Let $E$ be a regular level of the Hamiltonian $H$ with period $T$ and let $\psi$ be the normalized quasi-mode given in Proposition 5.3. Then

$$
\left\|\psi_{\hbar}\right\|^{2}=\frac{T}{2} \sqrt{2 \pi \hbar}+O\left(\hbar^{-1 / 2}\right) .
$$

5.1.2. Pairing formula. In this section we apply the explicit formulas of the preceding subsection to compute the desired scalar products.

Theorem 5.5. Let $\Sigma$ be either the once-punctured torus or the 4-times-punctured sphere, $\check{c}$ an admissible coloring of level $D$ and $\bar{r}$ an odd integer. Let $N=\bar{r} \Delta$ be the dimension of $V_{r}(\Sigma, \bar{r} \check{c})$ where $r=D \bar{r}$.

Let $\gamma_{0}$ and $\gamma_{1}$ be two curves on $\Sigma$. Denote by $T_{N}^{\gamma_{0}}$ and $T_{N}^{\gamma_{1}}$ the corresponding curve operators and by $H_{0}$ and $H_{1}$ the corresponding principal symbols (i.e. negatives of the trace functions).

Let $m_{0}$ and $m_{1}$ be two natural numbers and set for $i=0,1: \Sigma_{i}=\left\{z, H_{i}(z, \bar{z})=\right.$ $\left.-2 \cos \left(\frac{\pi m_{i}}{r}\right)\right\}$.

Suppose that $\Sigma_{0}$ and $\Sigma_{1}$ are non-empty regular curves which intersect transversally. Pick $z_{0} \in \Sigma_{0}$ and $z_{1} \in \Sigma_{1}$.

Let $\psi_{i} \in \mathcal{H}_{N}$ be a unit eigenvector of $T_{N}^{\gamma_{i}}$ with eigenvalue $-2 \cos \left(\frac{\pi m_{i}}{r}\right)$ and whose phase at $z_{i}$ is the same as the phase of $\left(\partial_{\bar{z}} H\left(z_{i}, \bar{z}_{i}\right)\right)^{-1 / 2}$ (notice that this condition defines $\psi_{i}$ up to a sign). Then,

$$
\left\langle\psi_{0}, \psi_{1}\right\rangle=\frac{ \pm 2 \hbar^{1 / 2}}{\sqrt{T_{0} T_{1}}} \sum_{z \in \Sigma_{0} \cap \Sigma_{1}} \frac{e^{-i \frac{\pi}{4} \operatorname{Sign}\left(\left\{H_{0}, H_{1}\right\}(z)\right)}}{\sqrt{\left|\left\{H_{0}, H_{1}\right\}(z)\right|}} e^{\frac{2 i \pi}{\hbar}\left(\int_{C_{0}} \eta-\int_{C_{1}} \eta\right)}+O\left(\hbar^{\frac{3}{2}}\right),
$$

where

- $\{.,$.$\} is the Poisson bracket on the sphere and \eta$ is the symplectic potential given in (39).

- For $i=0,1, C_{i}$ is a path in $\Sigma_{i}$ joining $z_{i}$ to $z$.

Remark 5.6. We can always assume that one of the two curve operators is the diagonal one. In this case all the eigenvectors, including the one corresponding to the extrema of the spectrum are given exactly by the WKB quasi-mode expression 
at leading order. Therefore the expression (40) is still valid in the case where $\Sigma_{0}$ is reduced to a single point. Note that in this case $\left\{H_{0}, H_{1}\right\}$ will be of order $\hbar^{\frac{1}{2}}$ in (and only in) the critical case where $d H_{0} \rightarrow 0$ at the two poles.

Proof. Let $T_{r}^{\gamma_{i}}, H_{i}, E_{i}, T_{i}, \psi_{i}, W_{i}, a_{i}$ be respectively the curve operator, the Hamiltonian, the level, the period, the quasi-mode, the phase function and the amplitude associated to each curve. Thanks to the normalization condition, the eigenvectors are close to the WKB quasi-modes $\psi_{i}=a_{i} e^{W_{i} / \hbar}$ as $\hbar$ goes to 0 . The proof comes from an estimation of the scalar product

$$
\left\langle\psi_{0}, \psi_{1}\right\rangle=\frac{i}{2 \pi} \int a_{0}(z) \overline{a_{1}(z)} e^{\frac{W_{0}(z)+\overline{W_{1}(z)}}{\hbar}} \frac{d z d \bar{z}}{\left(1+|z|^{2}\right)^{\frac{1}{\hbar}+1}} .
$$

From the Hamilton-Jacobi equation (34), the critical points of $F(z, \bar{z})=W_{0}(z)+$ $\overline{W_{1}(z)}-\log \left(1+|z|^{2}\right)$ are precisely the intersection points of the curves $\Sigma_{0}$ and $\Sigma_{1}$. Moreover for any $z \in \Sigma_{0} \cap \Sigma_{1}$ we compute

$$
\partial_{z}^{2} F=-\frac{\partial_{z} H_{0}}{\partial_{\bar{z}} H_{0}}\left(1+|z|^{2}\right)^{-2}, \partial_{\bar{z}}^{2} F=-\frac{\partial_{\bar{z}} H_{1}}{\partial_{z} H_{1}}\left(1+|z|^{2}\right)^{-2}, \partial_{z} \partial_{\bar{z}} F=-\left(1+|z|^{2}\right)^{-2} .
$$

By the stationary phase lemma, we get that the contribution to the pairing of a neighborhood of $z$ is given by

$$
\frac{a_{0}(z) \overline{a_{1}(z)}}{\pi\left(1+|z|^{2}\right)} 2 \pi \hbar \operatorname{det}\left(A_{1}\right)^{-1 / 2} e^{\frac{1}{\hbar}\left(W_{0}+\overline{W_{1}}-\log \left(1+|z|^{2}\right)\right)}
$$

where

$$
A_{\lambda}=\left(1+|z|^{2}\right)^{-2}\left(\begin{array}{cc}
2+\lambda\left(\frac{\partial_{z} H_{0}}{\partial_{\bar{z}} H_{0}}+\frac{\partial_{\bar{z}} H_{1}}{\partial_{z} H_{1}}\right) & i \lambda\left(\frac{\partial_{z} H_{0}}{\partial_{\bar{z}} H_{0}}-\frac{\partial_{\bar{z}} H_{1}}{\partial_{z} H_{1}}\right) \\
i \lambda\left(\frac{\partial_{z} H_{0}}{\partial_{\bar{z}} H_{0}}-\frac{\partial_{\bar{z}} H_{1}}{\partial_{z} H_{1}}\right) & 2-\lambda\left(\frac{\partial_{z} H_{0}}{\partial_{\bar{z}} H_{0}}+\frac{\partial_{\bar{z}} H_{1}}{\partial_{z} H_{1}}\right)
\end{array}\right) .
$$

Here $\operatorname{det}(A)^{-1 / 2}$ is defined for $\operatorname{Re}(A) \geq 0$ by analytic continuation from symmetric real and positive $A$. We compute $\operatorname{det}\left(A_{0}\right)^{-1 / 2}=\frac{1}{2}\left(1+|z|^{2}\right)^{2}$ and hence

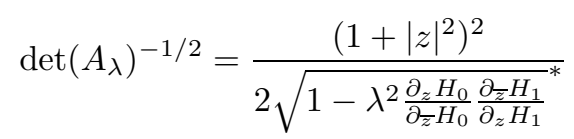

where the square root with the asterisk means the one with positive real part.

Using the formula $\left\{H_{0}, H_{1}\right\}=\frac{2 \pi}{i}\left(1+|z|^{2}\right)^{2}\left(\partial_{z} H_{0} \partial_{\bar{z}} H_{1}-\partial_{z} H_{1} \partial_{\bar{z}} H_{0}\right)$ and the fact that $a_{i}(z)=\frac{1}{1+|z|^{2}} \frac{1}{\sqrt{\partial_{\bar{z}} H_{i}}}$, we get:

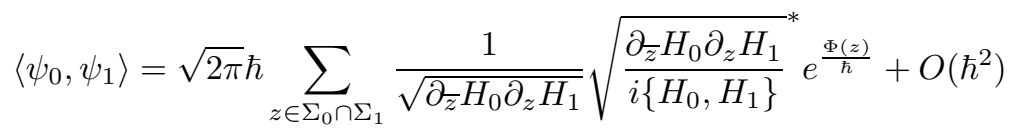

where we derive from (39) the following formula:

$$
\Phi(z)=W_{0}(z)+\overline{W_{1}}(z)-\log \left(1+|z|^{2}\right)=\frac{2 i \pi}{\hbar}\left(\int_{C_{0}} \eta-\int_{C_{1}} \eta\right) .
$$

We obtain the formula by dividing by the norm of $\psi_{0}$ and $\psi_{1}$. 


\subsection{Application to $6 \mathbf{j}$-symbols and punctured S-matrix.}

5.2.1. 6j-symbols. Consider the case where $\Sigma$ is a sphere with four punctures. Let $\gamma_{0}$ and $\gamma_{1}$ be respectively the curves $\zeta$ and $\eta$ shown in Figure 7 Let $\psi_{0}$ and $\psi_{1}$ be normalized eigenvectors of $T_{r}^{\gamma_{0}}$ and $T_{r}^{\gamma_{1}}$ with eigenvalues $-2 \cos \left(\frac{\pi m_{0}}{r}\right)$ and $-2 \cos \left(\frac{\pi m_{1}}{r}\right)$ as in Theorem 5.5. Then

$$
\left\langle\psi_{0}, \psi_{1}\right\rangle=u \frac{\sqrt{\sin \left(\frac{\pi m_{0}}{r}\right) \sin \left(\frac{\pi m_{1}}{r}\right)}}{\sin \left(\frac{\pi}{r}\right)}\left\{\begin{array}{lll}
\frac{a-1}{2} & \frac{b-1}{2} & \frac{m_{0}-1}{2} \\
\frac{d-1}{2} & \frac{c-1}{2} & \frac{m_{1}-1}{2}
\end{array}\right\}
$$

where $\left\{\begin{array}{lll}a & b & c \\ d & e & f\end{array}\right\}$ is the $6 \mathrm{j}$-symbol normalized as in [TW05] and $u$ is some complex number of modulus 1 .

Recall that the moduli space $\mathcal{M}\left(\Sigma, \frac{\pi a}{r}, \frac{\pi b}{r}, \frac{\pi c}{r}, \frac{\pi d}{r}\right)$ is diffeomorphic to the space of spherical quadrilaterals $\left(P_{1}, P_{2}, P_{3}, P_{4}\right)$ whose lengths are $l_{12}=\frac{\pi a}{r}, l_{23}=\frac{\pi b}{r}, l_{34}=$ $\frac{\pi c}{r}, l_{14}=\frac{\pi d}{r}$. The symbols of the operators $T_{r}^{\gamma_{0}}$ and $T_{r}^{\gamma_{1}}$ are $H_{0}=-2 \cos \left(l_{13}\right)$ and $H_{1}=-2 \cos \left(l_{24}\right)$. Moreover, the angle coordinates associated to the actions $l_{13}$ and $l_{24}$ are the exterior dihedral edges $\theta_{13}$ and $\theta_{24}$. In particular, one can write $\omega=\frac{r}{4 \pi^{2} N} d \theta_{13} \wedge d l_{13}$ (the normalization factor comes from the constraint $\left.\int \omega=1\right)$. We deduce from this formula that the period $T_{i}$ is equal to $\frac{r}{4 \pi N \sin \left(\frac{\pi m_{i}}{r}\right)}$. Let $G=\operatorname{det}\left(\cos \left(l_{i j}\right)\right)_{i, j=1}^{4}$. The following formula is proven in [TW05, Proposition 2.4.1]:

$$
\frac{\partial l_{i j}}{\partial \theta_{k l}}=-\frac{G^{1 / 2}}{\sin \left(l_{i j}\right) \sin \left(l_{k l}\right)} .
$$

We deduce from this that $\left\{H_{0}, H_{1}\right\}=\frac{16 \pi^{2} N}{r} G^{1 / 2}$. Moreover, following [TW05, the symplectic area $S$ of the region enclosed by $\Sigma_{0}$ and $\Sigma_{1}$ is equal to $\frac{r}{2 \pi^{2} N}\left(\sum_{a<b} l_{a b} \theta_{a b}-2 V\right)$ where $V$ is the volume of the spherical tetrahedron with length $l_{i j}$.

Let $z_{0}$ and $z_{1}$ be the two intersection points of the curves $\Sigma_{0}$ and $\Sigma_{1}$. Then, $\int_{C_{0}} \eta-\int_{C_{1}} \eta=S$. We deduce that

$$
\left\langle\psi_{0}, \psi_{1}\right\rangle=u \sqrt{\frac{4}{r} \sin \left(\frac{\pi m_{0}}{r}\right) \sin \left(\frac{\pi m_{1}}{r}\right)} \frac{\cos \left(\frac{S}{2}+\frac{\pi}{4}\right)}{G^{1 / 4}}+O\left(r^{-3 / 2}\right) .
$$

This gives an alternative proof of the following formula:

Proposition 5.7 ([TW05]).

$$
\left\{\begin{array}{lll}
\frac{a-1}{2} & \frac{b-1}{2} & \frac{e-1}{2} \\
\frac{d-1}{2} & \frac{c-1}{2} & \frac{f-1}{2}
\end{array}\right\}=\frac{2 \pi \cos \left(\frac{i r}{2 \pi}\left(\sum_{a<b} l_{a b} \theta_{a b}-V\right)+\frac{\pi}{4}\right)}{r^{3 / 2} G^{1 / 4}}+O\left(r^{-5 / 2}\right) .
$$

5.2.2. Punctured $S$-matrix. Let $\Sigma$ be the punctured torus and $\gamma$ and $\delta$ the curves shown in Figure 6. Let $\theta_{0} \in \mathbb{R} / 2 \pi \mathbb{Z}$ be the angle coordinate on $\mathcal{M}(\Sigma, \alpha)$ associated to $\tau_{0}=\arccos \left(\frac{1}{2} f_{\gamma}\right)$. By the constraint $\int \omega=1$ we get $\omega=\frac{1}{2 \pi(\pi-\alpha)} d \tau_{0} \wedge d \theta_{0}$ and from Lemma 3.2 we get $f_{\delta}=-2 \frac{\sqrt{\sin \left(\tau_{0}+\alpha / 2\right) \sin \left(\tau_{0}-\alpha / 2\right)}}{\sin \left(\tau_{0}\right)} \cos \left(\theta_{0}\right)=-2 \cos \left(\tau_{1}\right)$.

Define $H_{0}=f_{\gamma}, H_{1}=f_{\delta}$ and $T_{i}$ the period of the Hamiltonian flow of $H_{i}$. We compute $T_{i}=\left((\pi-\alpha) 2 \sin \left(\tau_{i}\right)\right)^{-1}$ and

$$
\left\{H_{0}, H_{1}\right\}=8 \pi(\pi-\alpha) \sin \left(\theta_{0}\right) \sqrt{\sin \left(\tau_{0}+\frac{\alpha}{2}\right) \sin \left(\tau_{0}-\frac{\alpha}{2}\right)} .
$$


Consider the intersection of the level sets $H_{0}=-2 \cos \left(\tau_{0}\right)$ and $H_{1}=-2 \cos \left(\tau_{1}\right)$. We have the symmetric formula:

$$
\left\{H_{0}, H_{1}\right\}=8 \pi(\pi-\alpha) \sqrt{\cos \left(\frac{\alpha}{2}\right)^{2}-\cos \left(\tau_{0}\right)^{2}-\cos \left(\tau_{1}\right)^{2}+\cos \left(\tau_{0}\right)^{2} \cos \left(\tau_{1}\right)^{2}}
$$

and hence we deduce from Theorem 5.5 the following proposition:

Proposition 5.8. Let $\Sigma$ be a punctured torus and let $\gamma_{0}$ and $\gamma_{1}$ be respectively the curves $\gamma$ and $\delta$ of Figure [6. Let $\psi_{0}$ and $\psi_{1}$ be normalized eigenvectors of $T_{r}^{\gamma_{0}}$ and $T_{r}^{\gamma_{1}}$ with eigenvalues $-2 \cos \left(\tau_{0}\right)$ and $-2 \cos \left(\tau_{1}\right)$ as in Theorem 5.5 where $\tau_{i}=\frac{\pi m_{i}}{r}$. Set

$$
G=\cos \left(\frac{\alpha}{2}\right)^{2}-\cos \left(\tau_{0}\right)^{2}-\cos \left(\tau_{1}\right)^{2}+\cos \left(\tau_{0}\right)^{2} \cos \left(\tau_{1}\right)^{2} .
$$

Then we have the following asymptotic formula:

$$
\left\langle\psi_{0}, \psi_{1}\right\rangle=\sqrt{\frac{8 \sin \left(\tau_{0}\right) \sin \left(\tau_{1}\right)}{r}} \frac{\cos \left(\frac{r}{2 \pi} \int_{\mathcal{D}} d \theta \wedge d \tau+\frac{\pi}{4}\right)}{G^{1 / 4}}+O\left(r^{-3 / 2}\right) .
$$

In this formula $\mathcal{D}$ is the domain of $\mathcal{M}(\Sigma, \alpha)$ defined by the equations $f_{\gamma_{0}} \geq$ $-2 \cos \left(\tau_{0}\right)$ and $f_{\gamma_{1}} \geq-2 \cos \left(\tau_{1}\right)$.

Let us deduce Result 5 from this proposition. Let $(\Gamma, c)$ be the colored graph given in Figure 1 where $c=\left(m_{0}, m_{1}, a\right)$. Then from TQFT axioms, denoting by $\Gamma_{i}$ the colored graphs shown in Figure 6, we have:

$$
\langle\Gamma, c\rangle=\frac{\left\|\Gamma_{0}|||| \Gamma_{1}\right\|}{\eta}\left\langle\psi_{0}, \psi_{1}\right\rangle
$$

where the first bracket stands for the Kauffman bracket and $\eta=\sqrt{\frac{2}{r}} \sin \left(\frac{\pi}{r}\right)$ is the quantum invariant of $S^{3}$. From formula (7), we get

$$
\frac{\left\|\Gamma_{0}|||| \Gamma_{1}\right\|}{\eta} \sim \frac{r^{3 / 2}}{\sqrt{2} \pi}\left(\frac{\left\langle m_{0}+\frac{a-1}{2}\right\rangle !\left\langle m_{0}-\frac{a+1}{2}\right\rangle !\left\langle m_{1}+\frac{a-1}{2}\right\rangle !\left\langle m_{1}-\frac{a+1}{2}\right\rangle !\left\langle\frac{a-1}{2}\right\rangle !^{4}}{\left\langle m_{0}\right\rangle !\left\langle m_{0}-1\right\rangle !\left\langle m_{1}\right\rangle !\left\langle m_{1}-1\right\rangle !\langle a-1\rangle !^{2}}\right)^{1 / 2}
$$

which proves Result [5], remarking that $\left\langle m_{i}\right\rangle=\sin \left(\tau_{i}\right)$.

5.3. Wick symbol and the sub-principal symbol. The aim of this section is to prove formula (2) from the asymptotic of the Wick symbol of the curve operators. Let $\sigma_{0}, \sigma_{1}$ be the first two terms in the expansion of the total Toeplitz symbols of the curve operator $T_{N}^{\gamma}$. From (12), we get

$$
W(z)=\frac{\left\langle T_{N}^{\gamma} \rho_{z}, \rho_{z}\right\rangle}{\left\langle\rho_{z}, \rho_{z}\right\rangle}=\sigma_{0}+\frac{1}{N}\left(\sigma_{1}+\Delta_{S} \sigma_{0}\right)+O\left(N^{-2}\right) .
$$

On the other hand, we have $T_{N}^{\gamma}=f_{0}(z, H)+\frac{1}{N} f_{1}(z, H)+O\left(N^{-2}\right)$. By a computation similar to Lemma 5.2 we get for a smooth function $g$ the expansion

$$
\begin{aligned}
\frac{g(H) \rho_{z_{0}}}{\rho_{z_{0}}}= & g\left(\frac{z \overline{z_{0}}}{1+z \overline{z_{0}}}\right)+\frac{1}{N}\left(-\frac{z \overline{z_{0}}}{1+z \overline{z_{0}}} g^{\prime}\left(\frac{z \overline{z_{0}}}{1+z \overline{z_{0}}}\right)\right. \\
& \left.+\frac{z \overline{z_{0}}}{2\left(1+z \overline{z_{0}}\right)^{2}} g^{\prime \prime}\left(\frac{z \overline{z_{0}}}{1+z \overline{z_{0}}}\right)\right)+O\left(N^{-2}\right) .
\end{aligned}
$$


This gives that the Wick symbol of $T_{N}^{\gamma}$ is up to $O\left(N^{-2}\right)$; the same as the one of the Toeplitz operator with symbol

$$
f_{0}\left(z_{0}, x_{0}\right)+\frac{1}{N}\left(f_{1}\left(z_{0}, x_{0}\right)-x_{0} \partial_{x} f_{0}\left(z_{0}, x_{0}\right)+\frac{1}{2} \frac{\left|z_{0}\right|^{2}}{\left(1+\left|z_{0}\right|^{2}\right)^{2}} \partial_{x}^{2} f_{0}\left(z_{0}, x_{0}\right)\right)
$$

where we have set $x_{0}=\frac{\left|z_{0}\right|^{2}}{1+\left|z_{0}\right|^{2}}$. We have $\sigma_{0}\left(z_{0}\right)=f_{0}\left(z_{0}, x_{0}\right)$ and from (36) , we get

$$
\frac{1}{2} \Delta_{S} \sigma_{0}\left(z_{0}\right)=f_{1}\left(z_{0}, x_{0}\right)-x_{0} \partial_{x} f_{0}\left(z_{0}, x_{0}\right)+\frac{1}{2} \frac{\left|z_{0}\right|^{2}}{\left(1+\left|z_{0}\right|^{2}\right)^{2}} \partial_{x}^{2} f_{0}\left(z_{0}, x_{0}\right)
$$

which implies that $\sigma_{1}=\frac{1}{2} \Delta_{S} \sigma_{0}$ and proves (2).

\section{Appendix A. Computations with fusion Rules}

Here is a toolkit to obtain the formula of Propositions 3.1 and 3.4 . There is a calculus for colored trivalent graphs in 3-space invented by Kauffman. Here we collect the formulas which are necessary for our computations where we set $[n]=\frac{A^{2 n}-A^{-2 n}}{A^{2}-A^{-2}}$. Here an edge colored by $n$ has to be interpreted as $n$ parallel copies of the same edge cabled by the Jones-Wenzl idempotent $f_{n}$. Be careful that the corresponding colored graph in TQFT has a shifted coloring and an alternate sign; see Subsection 2.5. We refer to MV94 for the proofs.
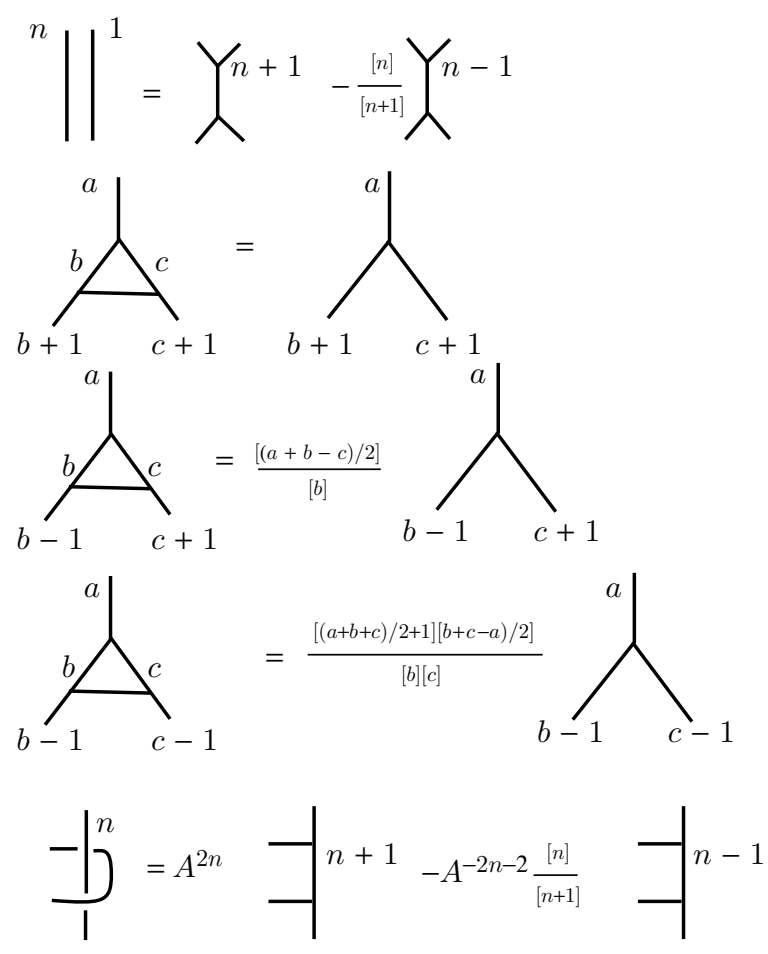

FiguRE 10. Fusion rules 


\section{REFERENCES}

[A06] Jørgen Ellegaard Andersen, Asymptotic faithfulness of the quantum SU $(n)$ representations of the mapping class groups, Ann. of Math. (2) 163 (2006), no. 1, 347-368, DOI 10.4007/annals.2006.163.347. MR2195137 (2007c:53131)

[A10] Jørgen Ellegaard Andersen, Toeplitz operators and Hitchin's projectively flat connection, The many facets of geometry, Oxford Univ. Press, Oxford, 2010, pp. 177-209, DOI 10.1093/acprof:oso/9780199534920.003.0010. MR2681692 (2012a:53177)

[A11] Jørgen Ellegaard Andersen, Hitchin's connection, Toeplitz operators, and symmetry invariant deformation quantization, Quantum Topol. 3 (2012), no. 3-4, 293-325. MR 2928087

[BHMV] C. Blanchet, N. Habegger, G. Masbaum, and P. Vogel, Topological quantum field theories derived from the Kauffman bracket, Topology 34 (1995), no. 4, 883-927, DOI 10.1016/0040-9383(94)00051-4. MR.1362791(96i:57015)

[BGPU] A. Bloch, F. Golse, T. Paul, and A. Uribe, Dispersionless Toda and Toeplitz operators, Duke Math. J. 117 (2003), no. 1, 157-196, DOI 10.1215/S0012-7094-03-11713-5. MR.1962785 (2003m:37121)

[BP00] Doug Bullock and Józef H. Przytycki, Multiplicative structure of Kauffman bracket skein module quantizations, Proc. Amer. Math. Soc. 128 (2000), no. 3, 923-931, DOI 10.1090/S0002-9939-99-05043-1. MR1625701(2000e:57007)

[C10] L. Charles, On the quantization of polygon spaces, Asian J. Math. 14 (2010), no. 1, 109-152, DOI 10.4310/AJM.2010.v14.n1.a6. MR2726596(2012a:53179)

[CM09] Laurent Charles and Julien Marché, Multicurves and regular functions on the representation variety of a surface in SU(2), Comment. Math. Helv. 87 (2012), no. 2, 409-431, DOI $10.4171 / \mathrm{CMH} / 258$. MR2914854

[CM11a] L. Charles and J. Marché, Knot state asymptotics I, AJ conjecture and abelian representations, arXiv:1107.1645

[CM11b] L. Charles and J. Marché, Knot asymptotics II, Witten conjecture and irreducible representations, arXiv: 1107.1646

[FWW] Michael H. Freedman, Kevin Walker, and Zhenghan Wang, Quantum SU(2) faithfully detects mapping class groups modulo center, Geom. Topol. 6 (2002), 523-539 (electronic), DOI 10.2140/gt.2002.6.523. MR1943758 (2004a:57037)

[G06] Răzvan Gelca, On the holomorphic point of view in the theory of quantum knot invariants, J. Geom. Phys. 56 (2006), no. 10, 2163-2176, DOI 10.1016/j.geomphys.2005.11.012. MR2241743 (2007d:53143)

[GU03] Răzvan Gelca and Alejandro Uribe, The Weyl quantization and the quantum group quantization of the moduli space of flat $\mathrm{SU}(2)$-connections on the torus are the same, Comm. Math. Phys. 233 (2003), no. 3, 493-512. MR.1962120(2003m:81120)

[G86] William M. Goldman, Invariant functions on Lie groups and Hamiltonian flows of surface group representations, Invent. Math. 85 (1986), no. 2, 263-302, DOI 10.1007/BF01389091. MR846929 (87j:32069)

[H90] N. J. Hitchin, Flat connections and geometric quantization, Comm. Math. Phys. 131 (1990), no. 2, 347-380. MR1065677 (91g:32022)

[Hu98] Johannes Huebschmann, Poisson geometry of flat connections for SU(2)-bundles on surfaces, Math. Z. 221 (1996), no. 2, 243-259, DOI 10.1007/PL00004249. MR.1376296 (97f:58029)

[MN08] Julien Marché and Majid Narimannejad, Some asymptotics of topological quantum field theory via skein theory, Duke Math. J. 141 (2008), no. 3, 573-587, DOI 10.1215/00127094-2007-006. MR2387432(2009a:57049)

[MP11] J. Marché and T. Paul, Toeplitz operators in TQFT via skein theory, arXiv:1108.0629 and hal-00612888, 2011.

[MV94] G. Masbaum and P. Vogel, 3-valent graphs and the Kauffman bracket, Pacific J. Math. 164 (1994), no. 2, 361-381. MR1272656(95e:57003)

[PU] Thierry Paul and Alejandro Uribe, A construction of quasi-modes using coherent states (English, with English and French summaries), Ann. Inst. H. Poincaré Phys. Théor. 59 (1993), no. 4, 357-381. MR 1254977 (95a:58126) 
[RT91] N. Reshetikhin and V. G. Turaev, Invariants of 3-manifolds via link polynomials and quantum groups, Invent. Math. 103 (1991), no. 3, 547-597, DOI 10.1007/BF01239527. MR 1091619 (92b:57024)

[TW05] Yuka U. Taylor and Christopher T. Woodward, $6 j$ symbols for $U_{q}\left(\mathfrak{s l}_{2}\right)$ and nonEuclidean tetrahedra, Selecta Math. (N.S.) 11 (2005), no. 3-4, 539-571, DOI 10.1007/s00029-005-0014-9. MR2215264(2007a:17027)

[TU91] Vladimir G. Turaev, Skein quantization of Poisson algebras of loops on surfaces, Ann. Sci. École Norm. Sup. (4) 24 (1991), no. 6, 635-704. MR1142906 (94a:57023)

[V] A. Voros. Thèse. Université d'Orsay, 1977.

[W89] Edward Witten, Quantum field theory and the Jones polynomial, Comm. Math. Phys. 121 (1989), no. 3, 351-399. MR990772(90h:57009)

Centre de mathématiques Laurent Schwartz (UMR 7640), Ecole Polytechnique, 91128 Palaiseau, France

Centre de mathématiques Laurent Schwartz (UMR 7640), Ecole Polytechnique, 91128 Palaiseau, France 